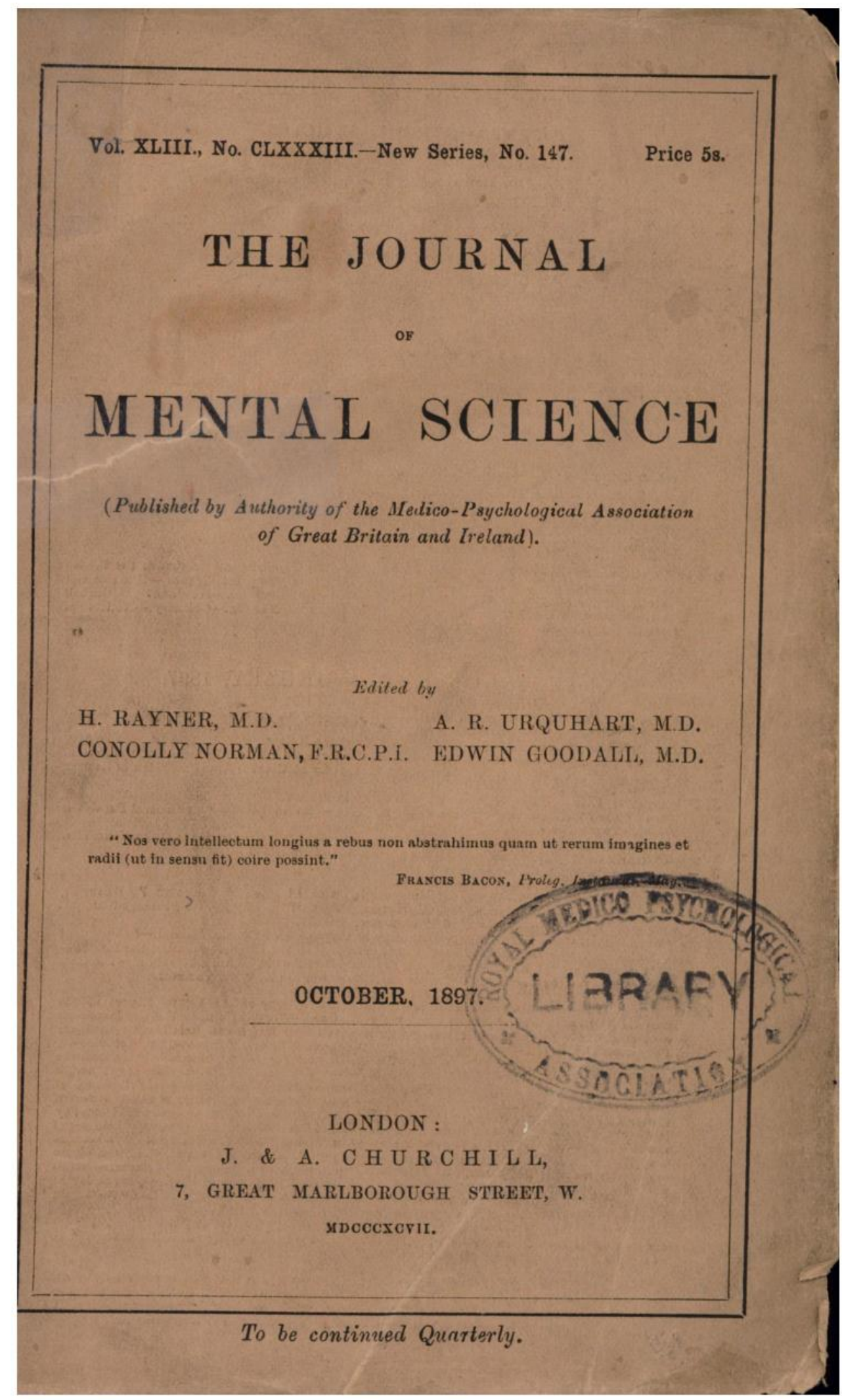


CONTENTS OF NO. 179.-OCTOBER, 1896.

\section{PART I.-ORIGINAL ARTICLES.}

W. Jullus Miokle, M.D.-Presldentlal Addreach

Georze R. Wlison, M.D.-The Significatice of Welsmenn's Doctrine in Invanity.

J. F. Briscoe, M.R.O.8,-Heredity in Mental Disence.

B. B. Stewart, M.D.-The Inerense of General Parwlyeis in England and Wales; Ita Cansation and Sigutiricences.

J. Macpherson, X.D.-The Hospital Treatnent of the Instne in Asylums.

A. R. Trurnbull, M.B.-Noto on temale Nurning in an Asylum 36ele Siek Hoom.

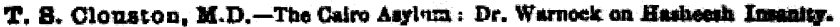

William W. Irelend, M.D.-Torquato Taswo and hle Blographers,

Olinionl Notes and Cases,-A Cave of Rocurrent Munia; by Joun G. Bavewoox, X.D.

Ocosstonal Notes of the Quarter.-The Annual Yeeting.-Rotitument of Dr. Desela--

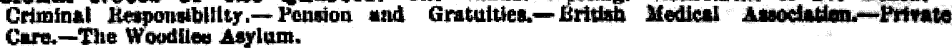

\section{PART II.-PSYCHOLOGICAL RETROSPECT.}

American Retrospect; by FLetcann Brach, U.D.

General Rotrofpect; by J. F. G. Pirresesax, M.R.C.8.

Crimina Anthropology ; by Havalock kitsa.

PART III.-NOTES AND NEWS.

Annual Meeting of the Medico-Psychological Amociation.-The Ruychological Section of the Brithin Medical Aserocintion.-liecent Medico-Legel Casts.-Parflumentary Intelligence.-Punslons and

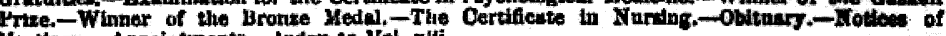
Moetings.-Appointments,-Index to Vol, xili.

CONTENTS OF No. 180.-JANUARY, 1897. PART I.-ORIGINAL ARTICLES.

W. Julins Wickle, M.D.-Atyoleil end Unutual Brain-Form, eapeclally in Belation to Mental Statns : A Study on Brutn-Surface Morplotory

W. Cilmore Fllis M.D.-Intah. A Mental Minidy of the Malay.

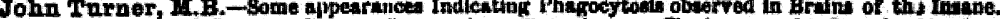

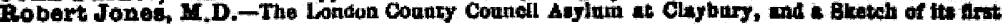
Working Yea

J. J. P1togirn. I.R.C.P. - The Detection of Insentty in Prtsons.

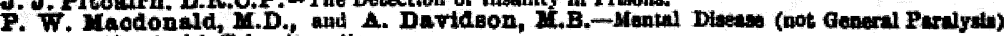
areciated with Trobes bortuits.

W. Ford Robertson, M.D. - Nute on Weigert's Theory regarding the Structure of the Nearoglia. Wilter Ohanning, M.D. - The Signifleance of Palatal Deformittes in latiota.

J. Ohristian $81 m p s 0$ n, M.D. -Ot Poat-Operntive Insenity.

H. Kornfold, M.D. On Muntial Auto-infectlon.

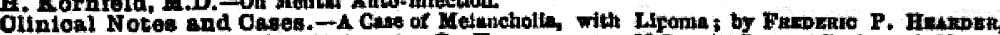
M.B.-Case of Cervicil Carles : by C. Hermaniwgrox, I, D. $-\mathbf{A}$ Case of Prolonged Hental Stupor anding in Recovery; by A. B. PAtrkesos, M.D.

Doosstonal Notes of the Quarter.-Patholngy th Asylums-A Bcottiak Proposal.-Independent Cntician upou Hitoloyical Appearances in the Brains of the Inaane.-Honsing the Intene.Suledes of " Unsound Mlind." -Insunity in Prisons, - Berl-Berf in Adchmond Aoylum, The Oare and Tralning of the Feeble-minded,-Nental Disenses in the New Nomenclinture.

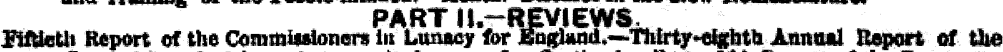

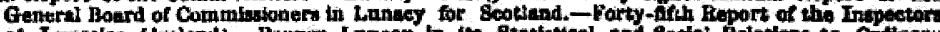

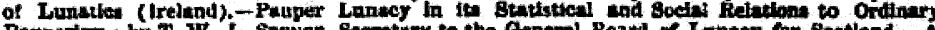
Panpertam, by T. W. L. Spxsice, Secretury to the Generw Boswd of Lainegy for Beotland. - A

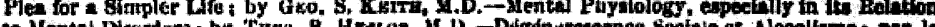

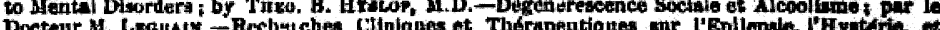

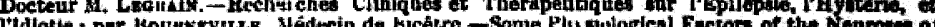

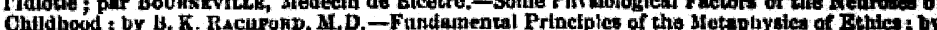

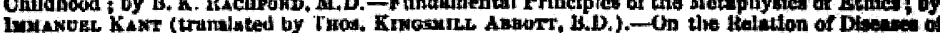

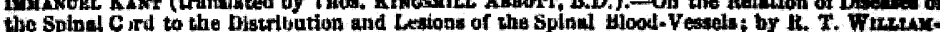

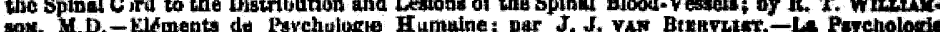

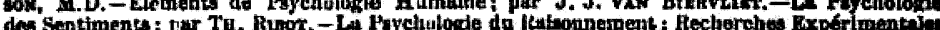

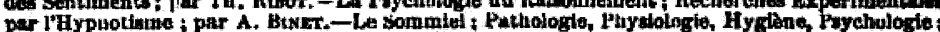

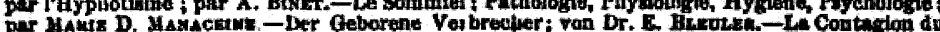

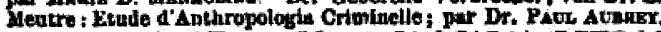

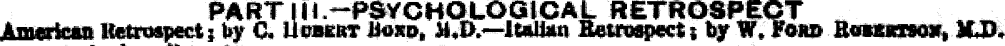
-Asylnm Hepart.

$$
\text { PART IV,-NOTES ANP NEWS }
$$

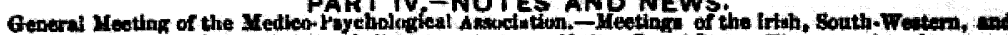

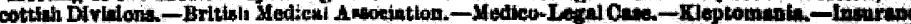

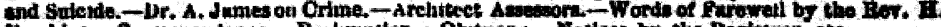

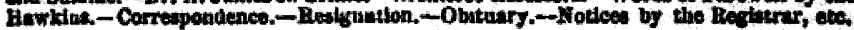




\title{
MEDICO-PSYCHOLOGICAL ASSOCIATION OF GREAT BRITAIN AND IRELAND.
}

\section{LIST OF CHAIRMEN.}

1841. Dr. Blake, Nottingham.

1842. Dr. de Vitré, Iancaster.

1849. Dr. Conolly, Hanwell.

1844. Dr. Thurnham, York Retreat.

1847. Dr. Wintle, Warneford House, Oxford.

1851. Dr. Conolly, Hanwell.

1852. Dr. Wintle, Warneford House.

\section{LIST OF PRESIDENTS}

\author{
1854. A. J. Sutherland, M.D., St. Luke's Hospital, London. \\ 1855. J. Thurnam, M.D., Wilts County Asylum. \\ 1856. J. Hitohman, M.D., Derby Connty Asylum. \\ 1857. Forbes Winslow, M.D., Sussex House, Hammersmith. \\ 1858. John Conolly, M.D., County Asylum, Hanwell. \\ 1859. Sir Charles Hastings, D.C.L \\ 1860. J. C. Bucknill, M.D., Devon County Asylum. \\ 1861. Joseph Lalor, M.D., Riohmond Asylum, Dublin. \\ 1862. John Kirkman, M.D., Saffolk County Agylum. \\ 1863. David Bkae, M.D., Royal Edinburgh Asylum. \\ 1864. Henry Munro, M.D., Brook Hozse, Clapton. \\ 1865. Wm. Wood, M.D., Kensington Honse. \\ 1866. W. A. F. Browne, M.D., Commissioner in Lunaoy for Sootland. \\ 1867. C. A. Lockhart Robertson, M.D., Haywards Heath Asylum. \\ 1868. W. H. O. Sankey, M.D., Sandywell Park, Cheltenham. \\ 1869. T. Laycook, M.D., Edinburgh. \\ 1870. Robert Boyd, M.D., County Asylum, Wells. \\ 1871. Henry Mandeley, M.D., The Lawn, Hanwell. \\ 1872. Sir James Coxe, M.D., Commissioner in Lanacy for Scotland. \\ 1873. Harrington Tuke, M.D., Manor House, Chiswick. \\ 1874. T. L. Rogers, M.D., County Asylum, Rainhill. \\ 1875. J. F. Duncan, M.D., Dublin. \\ 1876. W. H. Parsey, M.D., Warwiok County Asylum \\ 1877. G. Fielding Blandford, M.D., London. \\ 1878. J. Crichton-Browne, M.D., Lord Chancellor's Visitor. \\ 1879. J. A. Lush, M.D., Fisherton Honse, Salisbury. \\ 1880. G. W. Monld, M.R.C.S., Royal Asylum, Cheadle. \\ 1881. D. Hack Tuke, M.D., London. \\ 1882. W. T. Gairdner, M.D., Glasgow. \\ 1883. W. Orange, M.D., State Criminal Lunatic Asylum, Broadmoor. \\ 1884. Henry Rayner, M.D., County Asylum, Hanwell. \\ 1885. J. A. Eames, M.D., District Asylum, Cork. \\ 1886. Geo. H. Savage, M.D., Bethlem Royal Hospital. \\ 1887. Fred. Needham, M.D., Barnwood House, Gloncestor. \\ 1888. T. 8. Clonston, M.D., Royal Edinburgh Asylum. \\ 1889. H. Hayes Newington, M.R.C.P., Ticehurst, sussex. \\ 1890. Devid Yellowloes, M.D., Gartnavel Asylum, Glasgow. \\ 1891. E. B. Whitcombe, M.B.C.S., City Asylum, Birmingham \\ 1892. Robert Baker, M.D., The Retreat, York. \\ 1893. J. Murray Lindsay, M.D.; County Asylum, Derby. \\ 1894. Conolly Norman, F.R.C.P.I., Richmond Asylum, Dublin \\ 1895. David Nicolson, M.D., New Law Courts, Strand, W.C. \\ 1896. William Julius Mickle, M.D., Grove Hall Asylum, Bor.
}


THE

MEDICO-PSYCHOLOGICAL ASSOCIATION
OF GREAT BRITAIN AND IRELAND.

THE COUNCIL, 1897-98.

PEESIDENT. -T. W. MCDOWALL, M.D.

PRESIDENT-ELECT.-A. R. URQUHART, M.D.

MT-PRESIDENT.-W. J. MICKLE, M.D.

EMrRITUS TREASURER. -JOHN H. PAUL, M.D.

TREASURER. - H. HAYES NEWINGTON, M.R.C.P.Ed.

(HENRY RAYNER, M.D.

EDITORS OF JOURNAL $\left\{\begin{array}{l}\text { A. R. URQUHART, M.D. } \\ \text { CONOLLY NORMAN, F.R.C.P.I. }\end{array}\right.$

$\{$ EDWIN GOODALL, DI.D.

AUDITORS $\left\{\begin{array}{l}\text { E. B. WHITCOMBE, M.R.C.S. } \\ \text { T. SEYMODR TUKE, M.B. }\end{array}\right.$

DIVIBIONAL SECRETA RY FOR SCOTLAND.-A. R. TURNBULL, M.B.

DIVIBIONAI SECBETARY YOR IRELAND.-OSCAR T. WOODS, M.D.

DIVIBIONAL GEORETARY FOR NORTHERN \& MIDLAND DIVIBION -W. CROCHLE $P$ CLAPHAM, M.D.

DIVIGIONAL BEORETARY FOR BOUTH RABTERN DIVIBION.-E. W. WHITE, M.B. DIVIEIONAL BFCRETARY FOR SOUTH WESTREN DIVISION.-P. W. MACDONALD, M.D. GXNERAL BECRETARY.-ROBERT JONES, M.D., F.R.C.S.

geORETARY OF HDUCA TIONAL COMMITTER.-C. A. MERCIER, M.B.

REGISTRAR.-J. B. SPENCE, M.D.

MEMBERS OF COUNCIL.

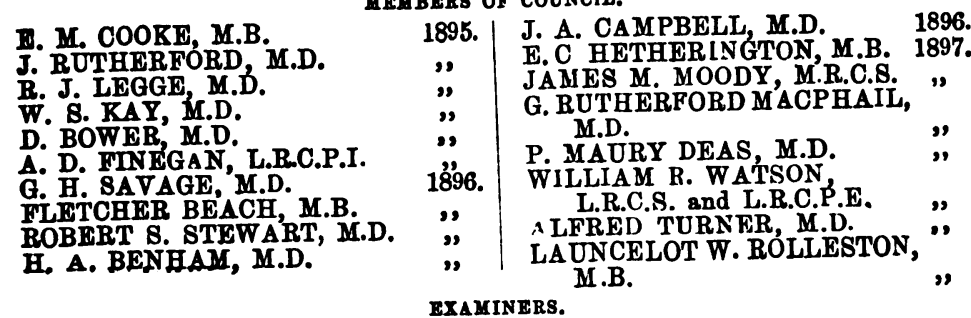

EXAMINERS.

WNGLAND $\left\{\begin{array}{l}\text { C. A. MERCIER, M.B. } \\ \text { R. PERCY SMITH, M.D. }\end{array}\right.$

SCOTLAND $\left\{\begin{array}{l}\text { J. RORIE, M.D. } \\ \text { CAMPBELL CLARK, M.D. }\end{array}\right.$

SOSCAR T. WOODS, M.D.

IRILAND \{ CONOLLY NORMAN, F.R.C.P.I.

PARLIAMRNTARY COMMITTRE.
DR. FLETCHER BEACH.
Dr. CONOLLY NORMAN.
DR. G. F. BLANDFORD.
18. D. M. CASSIDY.
DR. CLOUSTON.
DR. E. M. COOKE.
Mr. LEY.
DR. J. G. McDOWALL.
DR. MERCIFR.
DR. H. HAYES NEWINGTON.
DR. EVAN POWELL.
DR. SAVAGE.
DR. SAVRCY SMITH.
Dr. J. B. SPENCE.
DR. STOCKER.
DR. URQUHART.
DR. WIGLESWORTH.
DR. YELLOW LEES.
With power to add to their number. 


\section{HONORARY MEMBERB.}

1896. Allbutt, T. Cliford, M.D., F.R.C.P., Regius Profenacr of Phyic, Univ. Camb., St. Radegunds, Cambridge.

1881. Benedikt, Prof. M., Franciskaner Platz 5, Vienna.

1865. Biff, M., M.D., Editor of the Italian Journal of Mental Soience, 16, Borgo di Sall Colso, Milan.

1881. Brosius, Dr., Bendorf-Sayn, near Coblenz, Germany.

1876. Browne, Sir J. Crichton M.D. Edin., F.R.S.E., Lord Chancellor's Visitor, New Law Courts, Strand, W.C. (Prasidert, 1878.)

1887. Chapin, John B., M.D., Pennsylvania Hospital for the Insane, Phila. delphia, U.S.A.

1867.* Cleaton, John D., M.R.C.S. Eng., late Commissioner in Lunacy, 19, Whitehall Place, S.W.

1672. Courtenay, E. Maziere, A.B., M.B., C.M.T.C.D., M.D., Inspector of Junatics in Ireland, Lunacy Office, Dublin Castle. (Hon.

Member, 1891; Secretary for Ireland, 1876-1887.)
1884. Curwen, J., M.D., Warren, Pennsylvania State Hospital for the Insane, U.S.A.

1879. Echeverria, M. G., M.D.

1865. Falret, Jules, M.D., 114, Rue de Bac, Paris.

1892. Féré, Dr. Charles, 37, Boulevard Bt. Michel, Paris.

1895. Forrier, David, M.D., 34, Cavendish Square.

1872. Frasar, John, M.B., C.M., F.R.C.P.E., Commissioner in Lunacy, 19, Strathearn Road, Edinburgh.

- 1868. f Gairdner, W. T., M.D. Edin., F.R.S., Professor of Practice of Phyaie, 1888. 225, 8t. Vincent Street, Glasgow. (Presidernt, 1882.)

3886. Godding, Dr., Modical Superintendent, Government Hospital for Insane, Washington, U.S.

1875. Haughton, Rev. Professor S., School of Physic, Trinity College, Dublin, M.D.T.C.D., D.C.L. Oxon., F.R.S.

1881. Hughes, C.H., M.D., St. Louis, Missouri, United States.

1881. Krafft-Ebing, R. v., M.D., Vienna.

1866. Laehr, H., M.D., Schweizer Hof, bei Berlin, Editor of the Zeiteohrift für Psychiztrie.

1887. Lentz, Dr., Asile d'Aliénés, Tournai, Belgique.

1871. Manning, Froderick Norton, M.D. St. And., M.R.C.S. Eng., Inopec-

1884. tor of Asylums for New South Wales, Sydney.

1867. Moyer, Ludwig, M.D., University of Gottingen.

1881. Mierzojowski, Prof. I., Modico-Chirurgical Academy, 8t. Petersburg. 1866. Mitchell, Sir Arthur, M.D. Aberd., LL.D., K.C.B., late Commissioner

1871. in Lunacy for Scotland; 34, Drummond Place, Edinburgh.

1886. Morel, M. Jules, M.D., Hospice Guislain, Ghent.

1880. Motet, M., 161, Rue de Charonne, Paris.

1359. Neodham, Frederick, M.D. St. And., M.R.C.P. Edin., M.R.O.8. Fng., Commissioner in Lunacy, 19, Whitahall Place, 8.W. (Prusudxus, 1887.)

1891. O'Farrell, G. P., M.D., M.Ch. Univ. Dubl., Inspector of Lunatics in Ireland, 19, Fitewilliam Square, Dublin.

1881. Peeters, M., M.D., Gheel, Belgium. 
1873. Pitman, Sir Henry A., M.D. Cantab., F.R.C.P. Lond, Registrar of the Royal Colle;ze of Physiciaus, Enfield, Middlesex.

1886. Roussel, M. Théophile, M D., Sénateur, Paris.

1887. Schüle, Heinrich, M.D., Illenau, Baden, Germañ

1880. Sibbald, John, M.D. Edin., F.R.C.P. Edin., M.R.C.S. Eng., Commissioner in Lunacy for Scotland, 18, Greet King Street, Edinburgh. (Editor of Journal, 1871-72.)

1888. Stearns, H. P., M.D., The Retreat, Hartford, Conn., U.S.A.

1881. Tamburini, A., M.D., Reggio-Emilia, Italy.

1881. Virchow, Prof. R., University, Berlin.

1881. Voisin, A., M.D., 16, Rue Séguin, Paris.

1865. Wyatt, Sir William H., J.P., Chairman of Committee, County Asylum, Colney Hatch; 88, Rogent's Park Road.

\section{OORREDPONDINA MEMBERS.}

1896. Bianchi, Prof. Leonardo, Manicomio, Provinciale di Napoli.

1896. Bresler, Johannes, M.D., Irrenanstalt, Freiburg in Silesia, Germany. 1897. Buschan, Dr. G., Stettin, Germany.

1896. Cowran, F. M., M.D., 107, Perponcher Street, The Hague, Holland.

1880. Kornfeld, Dr. Herman, Grottkau, Silesia, Germany.

1889. Kowalowsky, Professor Paul, Kharkoff, Russia.

1895. Lindell, Emil Wilhelm, M.D., Gothenburg, Sweden.

1897. Näcke, Dr. P., Hubertusberg Asylum, Leipzig.

1886. Parant, M. Victor, M.D., Toulouse.

1890. Régis, Dr. E., 54, Rue Huguerie, Bordeaux.

1890. Ritti, Dr. J. M., Maison Nationale de Charenton, St. Maurice, Seine, France.

1893. Semılaigne, Réné, Dr., Secrétaire des Séances de la Société MédicoPsychologique de Paris, Avenue de Madrid, Nenilly, Seine, Paris. 
Alphabetical List of Members of the Association, with the year in which they joined. The Leterisk means Members who joined between 1841 and 1855.

1891. Adair, Thomas Stowart, M.B., C.M. Edin., Assistant Medical Officer, and Pathologist, Wadsley Asylum, near Sheffield.

1874. Adam. James, M.D. St. And., West Malling, Kent.

1845. Adam, Walter, M.D. Edin., Graham's Town Asylum, South Africa.

1868. Adums, Josiah O., M.D. Durh., F.R.C.S. Eng., Brooke House, U'pper Clapton, London.

1857. Adams, IRichard, I.R.C.P. Edin., M.R.C.S. Eng., Bodmin, Cornwall.

1880. Agar, S. H., L.R.C.P.I., Glendossil, Henley-in-Arden.

1886. Ayar, S. Hollingsworth, jun., B.A. Cantab., M.R.C.S , Glendossil, Henley-in-Arden.

189C. Alexander, Robert Reid, M.D. Aber., Medical Superintendent, Hanwell Lunatic Asylum.

1869. Aldridge, Chas., M.D. Aber., L.R.C.P., Plympton House, Plympton, Devon.

1893. Allan, Thomas Sprot, L.R.C.P. Edin., and L.M., L.R.C.S. Edin., etc., Queen's Road, Coventry.

1882. Alliott, A. J., M.D., Rosendal, Sevenoaks.

1885. Amsden, G., M.B., Medical Supt., County Asylum, Brentwood, Essex.

1888. Anderson, W. A., M.B., Bucks County Asylum. Stone, Aylesbury.

1894. Andriezen, W. Lloyd, M.D. Lond., Darenth Asylum, Dartford, Kent.

1894. Angus, Charles, M.B., C.M., Senior Assistant Physician, Royal Asylum, Aberdeen.

1887. Aplin, A., M.R.C.S.E. and L.R.C.P. Lond., Medical Superintendent, County Asylum, Snenton, Nottingham.

1892. Atherstone, Walter H., M.D., Surgeon-Superintendent, Port Alfred Asylum, South Africa.

1875. Atkins, Ringrose, M.A., M.D. Queen's Univ. Ire., Medical Superintendent, District Asyium, Waterford.

1891. Aveline, Henry T. S., M.R.C.S., L.R.C.P., M.P.C., Medical Superintendent, County Asylum, Cotford, pear Taunton, Somerset.

1894. Baily, Percy J., M.B. Edin., Senior Assistant Medical Otficer, London County Asylum, Hanwell, W.

1878. Baker, H. Morton, M.B. Edin., Assistant Medical Officer, Leicester Borough Asylum, Leicester.

1888. Baker, John, M.B., 471, Caledonian Road, London, N.

1876. Baker, Robert, M.D. Edin., Visiting Physician, The Retreat, York (Pressident, 1892), 41, The Mount, York.

1378. Barton, James Edwazd, L.R.C.P. Edin., L.M., M.R.C.S., Medical Supt., Surrey County Lunatic Asylum, Brookwood, Woking.

1895. Barraclough, Herbert, M.B., County Asylum, Devizes.

1864. Bayley, J., M.R.C.S., Medical Supt., Lunatic Hospital, Northampton.

1893. Bayley, Joseph Herbert, M.B., C.M. Edin., Assistant Medical Officer, St. Andrew's Hospital, Northampton.

1874. Beach, Fletcher, M.B., F.R.C.P. Lond., formerly Medical Suporintendent, Darenth Asylum, Dartford ; Winchester House, Kingston Hill, Surrey, and 64, Welbeck Street, W. (Gen. Secretary, 1889-1896.)

1897. Beadle, T. Alfred, L.R.C.P., I.R.C.S. Edin., Assistant Medical Officer, District Asylum, Hartwood, Glasgow.

1892. Beadles, Cecil F., M.R.C.S., L.R.C.P., Assistant Medical Officer, Colney Hatch Asylum.

1896. Besmish, George, L.R.C S.I., L.R.C.P.E., I.M., Medical Officer, H.M. Prison, Jiverpool. 
1872. Benham, H. A., M.D., Medical Superintendent, City and County Asylum, Stapleton, near Bristol.

1894. Bernand, Dr. Walter, District Asylum, Londonderry.

1864. Bigland, Thomas, M.R.C.8. Eng., L.S.A. Lond., Bigland Hall, Backbarrow, near Ulverston, Lancashire.

1894. Blachford, James Vincent, M.B., B.S. Durham, Assistant Medical Officer, Bristol Asplum, Fishponds, near Bristol.

1683. Blair, Robert, M.D., Medical Superintendent, Woodileo Asylum, Lenzie, near Glasgow.

1879. Blanchard, E. S., M.D., Medical Superintendent, Hospital for Insano, Charlotte Town, Prince Edwrard's Island.

1857. Blandford, George Fielding, M.D. Oxon., F.R C.P. Lond., 48, Wimpole Street, W. (Prksidrnt, 1877.)

1897. Blandford, Joseph John Guthrie, B.A., D.P.H. Camb., M.R.C.S. Eng., L.R.C.P. Lond., Assistant Medical Officer, London County Asvlum, Banstead, Surrey.

1888. Blaxland, Herbert, M.R.C.S. Modical Superintendent, Callan Park Asylum, New South Wales.

1090. Blumer, G. Alder, M.D., Medical Superintendent of the State Hospital for the Insane, Utica, N.Y., U.S.A.

1895. Bodington, George Fowler, M.D. Durh., F.R.C.8. Eng, M.R.C.P. Lond., Medical Superintendent, Government Asylum for the Insane, Province of British Columbia, Canada

1E96. Boddie, William, M.B., C M. Aber., Assistant Medical Officer, Fisherton House, Salisbury.

1892. Bond, Charles Hubert, B. Sc., M.D., Ch.M. Edin., London County Asylum. Banstead, Sutton, Surrey.

1877. Bower, David, M.D. Aberd., Springfield House, Bedford

1877. Bowes, John Ireland, M.R.C.S. Eng., L.S.A., Medical Superinten. dent. County Asylum. Devizes, Wilts.

1893. Bowos, William Henry, M.D. Lond., Assistant Medical Officer, Plymouth Bo:nugh Asylum, Ivybridge, Devon

1896. Boycott. A. N.. M.D. Lond., M.R.C.S. Eng., I, R.C.P. Lond., Assist. Med. Officer, London County Asylum, Canehill, Purley, Surrey.

1683. Boys, A. H. L.R.C.P. Edin., Chequer Lawn. St. Albans.

1894. Bradburn, James Denham, I.R.C.P., F.R.C.S. Edin., Fair Home, Leamington.

1891. Braine-Hartnell, George, I, R.C.P. Lond., M.R.C.S. Fng., Senior Assistant Medical Officer, County and City Asylum, Powick, Worcester.

1893. Bramwell, John Milne. M.B., C.M. Edin., 2, Henrietta Street, Cavendish Square, London, $\mathbf{W}$.

1881. Brayn. R., L.R.C P. Lond., Medical Superintendent, Broadmoor Asylum. Crowthorne, Berks.

1892. Bristowe, Hubert Carpenter, M.D. Lond., Wrington R S.O., Somerset.

1895. Brisooe, Jehn Frederick. M.R.C.S. Fng., Resident Medical Superintendent, Westbrooke H(ruse Asvlum, Alton, Hants.

1864. Brodie, David, M.D St. And., L.R.C.S. Edin., 12, Patten Road, Wandsworth Common, S.W.

1891. Bruce. John, M.B., C.M. Edin., M.P.C., Lauriston Town Halı Square, Grimsby.

1893. Bruce. Lewis C.. M.B. Edin., Assistant Medical Officer, Morningside Asvlum. Edinburgh.

1893. Brunton. Walter Revncr, M.B. Durh., 19, Queen's Road, Twickenham.

- Brushfield, Dr., Budleigh Salterton, Devon.

1896. Bubb. William. M.R.C.S. J R.C.P. Innd.. Second Assistant Medical Officer, Worcester County Asylum. Powick, near Worcester.

1894. Buggy, Louis, L.R.C.S.I., L.M., L.R.C.P.I., Assistant Modical Officer, District Asylum, Kilkenny. 
1892. Bullen, Frederick St. John, M.R.C.S. Eng., 10, Pembroke Road, Clifton, Bristol.

1890. Burke, John R., M.D., Deputy Inspector General of Hospitals and Fleets (retired); late Assistant Medical Officer Central Criminal Asylum, Dundrum, Co. Dublin, Ireland, 22, Gardiners Placo, Dublin.

1869. Burman, Wilkio J., M.D. Edin., Ramsbury, Hungerford, Berks.

1891. Caldecott, Charles, M.B., B.S. Lond., M.R.C.S., Medical Superintendent, Earlswood Asylum, Redhill, Surrey. .

1889. Callcott, J. T., M.D., Medical Suparintendent, Borough Asylum, Newcastle-on-Tyne.

1894. Campbell, Alfred Walter, M.D. Edin., Pathologist, County Asylum, Rainhill, near Prescott, Lancashire.

1857. Campbell, John A., M.D. Glas., Medical Superintendent, Cumberland and Westmorland Asylum, Garlands, Carlisle.

1897. Campbell, Keith, M.B. Edin., Assistant Medical Officer, District Asylum, Murthly, Perth, N.B.

1880. Campbell, P. E., M.B., C.M., Senior Assistant Medical Officer, District Asylum, Caterham.

1897. Camplell, Robert Brown, B.B., C.M. Edin., Assistant Medical (Oficer, Jistrict Asylum, Inverness.

1890. Cameron, James, M.B., C.M. Edin., 13, Fettes Row, Edinburgh.

1874. Cameron, John, M.D. Edin., Medical Superintendent, Argyll and Bute Asylum, Lochgilplead.

1897. Cappe, Herbert Nelson, M.R.C.S. Eng., L.R.C P. Lond., Assistant Medical Officer, Surrev County Asylum, Brookrood.

1896. Cardale, Henry Jaspar, M.B., C.M. Edin., Assistant Medical Officer, Fisherton House, Salisbury.

1891. Carswell, John, J.K.C.P. Fdin., L.F.P.S. Glasg., Certifying Medical Oftice:, Bar.ony Parish, 5, Roval Crescent, Glasgow.

1881. Case, J., M.R.C.S., Medical Superintendent, Leavesden, Herts.

1896. Cashman, James, M.B., B.Ch., B.A.O. Royal Univ. Ire., Assistant Medical Officer, Cork District Asylum.

1896. Cassidy, Cha!les S., M.13. Edin., Assistant Medical Officer, Grabamstown. South Africa.

j874. Cassidy, D. M.. M.D., C.M. McGill Coll., Montreal. D.Sc (Public Health), Edin., F.R.C.S. Edin., Medical Superintendent, County Asylum, Lancaster.

1888. Chambers, James, M.D., M.P.C. The Priory, Roehampton.

1865. Chapman, Thomas Algernon, M.D. Glas., I..R.C.S. Edin. ; lato Hereford County .aná City Asylum, Hereford, Elmscroft, Elms Road, Redhill, Surrev.

1880. Christia, J. W. Stirling. M.D., Mer. Supt., Countr Asvlum, Stafford.

1878. Clap!ıam, Wm. Crochlev S., M.D., M.R.C.P., The Grange, Rotherham.

1863. Clapton, Edward, M.D. Iond., F.R C.P. Lond., late Physician, St. Thomas's Hospital, late Visitor of Lunatics for Surrey; 22, St. Thomas's Street, Borough, S.E.

1879. Clark, Archibald C., M.D. Edin., Melieal Superinteudent, I.anarkshire Asylum, Hartwood, Shotts, N.B.

1879. Clarke, Henry, J.R.C.P. Jond.. H.M. Prison, Wakefield.

1662. Clouston, T. S., M.D. Edin., F.R.C.P. Edin., F.R.S.E., Physician Superintendent, Royal Asylum, Morningside, Fdinburgh. (Editor of Journal, 1873-1881.) (Presinent, 1888.)

1879. Cobbold, C. S. W., M.D., Bailbrook House, Bath.

1892. Cole, Robert Henry, M D. Lond., M.R.C.P. Lond., 53, Upper Berkeley Sireet, W.

1896. Coles, Richard Ambrose, The Yews, Ledbury.

1896. Collins, George Fletcher, M.R.C.S. Eng., L.K.C.P.L., D.P.H. Cantab., 3, Windsor Terrace, Penarth, near Cardiff. 
1888. Cones, John A., M.R.C.S., Burgess Hill, Sussex.

1895. Conry, John, M.D. Aber., Fort Beaufort Asylum, South Africa.

1878. Cooke, Edward Marriott, M.B., M.R.C.S. Eng., Medical Superintendent, County Asylum, Worcester.

1891. Corner, Harry, M.B. Lond., M.R.C.S., L.R.C.P., M.P.C., Brooke House, Soathgate, $\mathbf{N}$.

1891. Cowan, John J., M.B., C.M. Edin., Leigh Sinton, Malvern.

1893. Cowen, Thomas Phillips, M.B., B.S. Lond., Assistant Medical Officer, County Asylum, Prestwich, Manchester.

1684. Cox, L. F., M.R.C.S., Medical Supt., County Asylum, Denbigh.

1878. Craddock, F. H., B.A. Oxon., M.K.C.S. Eng., I.S.A., Medical Superintendent, County Asylum, Gloucester.

1892. Craddock, Simuel, M.î.C.S. Eng., South Hill House, Bath.

1893. Craig, Maurice, M.A., M.B., B.C. Cantab., M.R.C.P. Lond., Assistant Medical Officer, Bethlem Royal Hospital, Southwark.

1894. Crawford, Cyril R., M.R.C.S. Eng., L.R.C.P. Loud., Sussex County Hospital, Brighton.

1897. Cribb, Harry Gifford, M.R.C.S. Eng., L.R.C.P. Lond., Assistant Medical Officer, London County Asylum, Canehill, Surrey.

1694. Cullinan, Henry M., L.R.C.P.I., L.R.C.S.I., Second Assistant Medical Officer, Richmond District Asylum, Dublin.

1869. Daniel, W. C., M.D. Heidelb., M.R.C.S. Eng., Epsom, Surrey.

1896. Davidson, Andrew, M.B., C.M. Aber., Assistant Medical Officer, County Asylum, Dorchester.

1868. Davidson, John H., M.D. Edin., Delamere House, Liverpool Road, Chester.

1874. Davies, Francis P., M.D. Edin., M.R.C.S. Eng., Kent County Asylum, Barming Heath, near Maidstone.

1891. Davis, Arthur N., L.R.C.P., L.R.C.S. Edin., Medical Superintendent, Borough Asylum, Ivybridge, Devon.

1894. Dawson, William R., B.Ch., B.A.O. Univ. Dubl., Assistant Medical Supt., Farnham House Private Asylum, Finglas, Dublin.

1869. Deas, Peter Maury, M.B. and M.S. Lond., Medical Superintendent, Wonford House, Exeter.

1896. Dewar, Margaret C., M.B., C.M. Univ. Glasg., Gartnavel Royal Asylum, Glasgow.

1876. Dickson, F. K., F.R.C.P. Edin., Wye House Lunatic Asylum, Buxton, Derbyshire.

1879. Dodds, William J., M.D.. D.Sc. Edin., Valkenberg, Mowbray, near Cape Town, South A frica.

1886. Donaldson, R. Lockhart, A.B., M.B., B.Ch. Univ. Dub., M.B., M.P.C., Assist. Medical Officer, District Asylum, Monaghan.

1889. Donaldson, William Ireland, B.A., M.B., B.Ch., Univ. Dublin, Assistant Medical Officer, London County Asylum, Canchill, Purley, Surrey.

1892. Donelan, J. O'C., L.R.C.P.I., L.R.C.S.I., M.P.C., First Assistant Medical Officer, Richmond District Asylum, Dublin

1891. Douglas, Archibald Robertson, L.R.C.S., L.R.C P. Edin., The Grove, Portland, Dorset.

1890. Douglas, William, M.D. Queen's Univ. Irel., M.R.C S. Eng., Medical Officer, Provident Dispensary, Leamington Spa; Dalkeith House, 7, Clarendon Place, Leamington Spa.

1897 Dove, Emily Louisa, M.B. Lond., Assistant Medical Officer, London County Asylum. Clavbury, Essex.

1884. Drapes, Thomas, M.B., Medic3l Superintendent, District Asylum, Enniscorthy, Ireland.

3895. Drury, Arthur. M.B.. C.M. Edın., Medical Officer, Halifax Union, Landois House, Halifax. 
1874. Eager, Reginald, M.D. Lond., M.R.C.S. Eng., Northwoods, near Bristol.

1873. Eager, Wilson, L.R.C.P. Lond., M.R.C.S. Eng.

1888. Farle, Leslie, M.D. Edin., 21, Gloucester Place, Hyde Park, W.

1891. Earls, James Henry, M.D., M.Ch., etc., 71, Brighton Square, Dublin.

1895. Easterbrook, Charles C., M.A., M.B., C.M., Assistant Medical Officer, Royal Asylum, Edinburgh.

1862. Eastwood, J. William, M.D. Edin., M.R.C.P. Lond., Dinsdale Park, Darlington.

1895. Edgerly, Samuel, M.B., C.M. Edin., Assistant Medical Officer, Roxburgh District Asylum, Melrose.

1897. Edwards, Francis Henry, M.D. Brux., L.R.C.P. Lond., M.R.C.S. Eng., Assistant Medical Oificer, Camberwell House, S.E.

1889. Elkins, Frank A., M.13., C.M. Edin., M.P.C., Medical Superinten. dent, Sunderland Borough .Asylum.

1873. Elliot, G. Stanley, M.R.C.P. Edin., F.R.C.S. Edin., Medical Superintendent, Caterham, Surrey.

1890. Ellis, William Gilmore, M.D. Brux., Superintendent, Government Asylum, Singaporo.

1895. Eurich, Frederick William, M.B., C.M. Edin., Pathologist, County Asylum, Whittingham, Preston.

1861. Eustace, J., M.D. Trin. Coll. Dub., L.R.C.S.I., Highfield, Drumcondra, Dublin.

1894. Eustace, Henry Marcus, M.B., B.Ch., B.A. Univ. Dublin, Assistant Physician, Eampstead and Highfield Private Asylum, Glasnevin,

1897. Everett, William, M.D., Assistant Medical Officer, County Asylum, Chartham Downs, Kent.

1891. Ewan, John Alfred, M.A., M.B., C.M. Edin., M.P.C., Medical Superintendent, Kesteven and Grantham District Asvlum.

1884. Ewart, C. T., M.B., C.M. Aberd., Assistant Medical Officer, Colney Hatch Asylum, Middlesex.

1896. Ewbank, Arthur George, M.R.C.S., L.R.C.P. Lond., Assistant Medical Officer, Middlesex County Asylum, Tooting, London, 8.W.

1888. Ezard, E. H., M.D., D.Sc. Edin., M.P.C., 220, Lewisham High Road, St. John's, S.E.

1894. Farquharson, William F., M.B. Edin., Assistant Medical Officer, Counties Asylum, Garlazds, Carlisle.

1892. Farquharson, Alexander Charles, M.D., M.C., D.P.H. Camb., Senior Assistant Medical Officer, Burntwood Asylum, Burntwood, near Lichfield.

1895. Felvus, Charles Percival, L.R.C.P. and L.R.C.S. Edin., L.F.P.S. Glasg., care of Messrs. Harris and Copy, 41 and 42, Hatton Garden, London.

1897. Fielding, James, M.D. Victoria Univ. Canada,, M.R.C.S. Eng., L.R.C.P. Edin., Medical Supt., Bethel Hospital, Norwich.

1867. Finch, W. Corbin, M.R.C.S. Eng., Fisherton House, Salisbury.

1877. Finch, John E. M., M.D., Medical Supt., Borough Asylum, Leicester.

1889. Finch, Richard T., B.A., M.B. Cantab., Resident Medical Officer, Fisherton House Asylum, Salisbury.

1890. Findlay, George, M.B., C.M. Aber., Brailes, Shipston-on-Stour.

1882. Finegan, D. O'Connell, L.R.C.P.I., Medical Superintendent, District Asylum, Mullingar.

1889. Finlay, Dr., County Asylum, Bridgend, Glamorgan

1891. Finny, W. E. St. Lawrence, M.B. Univ. Irel., Kenlis, Queen's Road, Kingston Hill, Surrey.

1888. Fitzgerald, G. C., M.B., B.C. Cantab., M.P.C., Medical Superintendent, Kent County Asylum, Chartham, near Canterbury.

1894. Fitzgerald, Charles E., M.D., F.R.C.S.I., Surgeon Oculist to the Queen in Ireland, 27, Üpper Merrion Street, Dublin. 
1872. Fletcher, Robert Vicars, Esq., F.R.C.S.I., L.R.C.P.I., L.R.C.P. Edin., Medical Supt., District Asylum, Ballinasloo, Ireland.

1894. Fleury, Eleonora Lilian, M.D., B.Ch., R.U.I., Assistant Medical Officer, Richmond Asylum, Dublin.

1880. Fox, Bonville Bradley, M A. Oxon., M.D., M.R.C.S., Brislington House, Bristol.

1861. Fox, Charles H., M.D. St. And., M.R.C.S. Eng., 35, Heriot Row, Edinburgh.

1897. Fox, George Aubrey Townsend, M.R.C.S. Eng., L.R.C.P. Lond., Assistant Merical Officer, Ccunty Asylum, Chartham Downs, Kent.

1896. Francy, Eric, M.B., B.S. Durh., Assistant Medical Officer, Northumberland County Asplum, Morpeth.

1881. Fraser, Donald, M.D., 3, Orr Square, Paisley.

1873. Garner, W. H., Esq., F.R.C.S.I., A.B.T.C.D., Medical Superintendent, Clonmel District Asylum.

1893. Garth, H. C., M.B., C.M. Edin., 4, Harrington Street, Calcutta, India. 1867. Gasquet, J. R., M.B. Lond., St. George's Rotreat, Burgess Hill, and 1, College Gate, Brighton.

1890. Gaudin, Francis Neel, M.R.C S., I.S.A., M.P.C., Medical Superintendent, The Grove, Jersey.

1885. Gayton, F. C., M.D, Brookwoorl Asylum, Surrey

1896. Geddes, Join W., M.B., C M. Edin., Assistant Medical Officer, Durham County Asylum, Winterton, Ferryhill, Durham.

1871. Gelston, R. P., I.R.C.P.I. L.R.C.S.I., Medical Superintendent, District Asylum, Ennis, Ireland.

1892. Gemmel, James Francis, M.B. Glasg., Assistant Medical Officer, County Asjlum, Lancaster.

1889. Gibbon, William, L.R.C.P.I., L.F.P.S. Glasg., Senior Assistant Medical Officer, Joint Counties Asylum, Carmarthen.

1889. Gill, Dr. Stanley, B.A., M.D., M.R.C.P̌. Lond., Shaftesbury House, Formby, Lancashire.

1897. Gilmour, John Rutherford, M.B., C.M. Edin., Assistant Physician, Crichton Royal Institution, Dumfries.

1878. Glendinning, James, M.D. Glosg., L.R.C.S. Edin., I.M., Medical Superintendent, Joint Counties Asylum, Abergavenny.

1892. Goldie, E. Milliken, M.B., C.M. Edin.. Poplar and Stepney Sick Asvlum, Devons Road, Bromley, London, E.

1697. Good, Thomas Saxty, M.R.C.S. Eng., I.R.C.P. Lond., Assistant Medical Officer, County Asylum, Littlemore. Oxford

1889. Goodall, Edwin. M.D., M.S. I.ond., M.P.C., Merical Superintendent, Joint Counties Asylum, Carmarthen. (Editor of Journa!.)

Gordon, W. S., M B., District Asvlum, Mullingar.

1893. Gordon-Munn, John Gordon, M.B., C.M. Edin., Assistant Medical Officer, The Hall, Bushey. Herts.

1888. Graham, T., M.D. Glasg., 3, Garthland Place, Paisley.

1894. Graham, Samuel, L.R.C.P. Lond., Assistant Medical Officer, District Asvlum, Belfast.

1887. Graham, W., M.D. (R.U.I.), Medical Superintendent, District Lunatic Asylum, Belfast.

1890. Grimshaw, Farbrace Sidney, M.D. L.R.C.P. Irel., I, R.C.S. Edin., L.M., I.A.H. Dubl., The Villa. Stillington. Yorkshire

1897. Grant-Wilson. Charles Westbrook, J.R.C.P. Lond., M.R.C S. Eng., Heathfield Hoisso, Streatham Common.

1891. Greatbatch, Herbert W, M.B., C.M. Edin., 30, Bridge Street, Montrose, N.B.

1886. Greenlees, T. Duncan, M.B., Medical Superintendent to the Grabamstown Asylum, Cape of frood Hope. 
1896. Greane, Thomas Adam, Assistant Medical Officer, District Asylum, Ennis, Iroland.

1894. Griffin, Edward W., M.D., M.Ch., R.W.I., Assistant Medical Officer, The Asylum, Killarney.

1896. Griffiths, George Baths, M.R.C.S., L.R.C.P. Lond., Assistant Surgeon, H.M. Con vict Prison, Portland.

1886. Grubb, J. Strangman, L.R.C.P. Edin., North Common, Ealing, W.

1879. Gwynn, S. T., M.D., St. Mary's House, Whitechurch, Salop.

i894. Gwynn, Charles Henry, M.D. Edin., Co-Lirensee, St. Mary's House, Whitechurch, Salop.

1888. Habgood, W., M.D., L.R.C.P., Tower Croft, Marple, Cheshire.

1866. Hall, Edward Thomas, M.R.C.S. Eng., Newlands House, Tooting Beck Road, Tooting Common, Chelsea, S.W.

1394. Halstead, Harold Cecil, M.D. Durh., Assistant Mcdical Officer, Peckham House, Peckham.

1896. Hanbury, William Reader, Assistant Medical Officer, Ccunty Asylum, Dorchester, Dorset.

1375. Harbinson, Alexander, M.D. Irel., M.R.C.S. Eng , Assistant Medical Officer, County Asylum, Iancaster.

1895. Harpar, Thomas Edward, L.R.C.P. Lond., M.R.C.S. Eng., Assistan Sedical Officer, St. Ann's Heath. Virginia Water.

1897. Harris. William, M.D St. And., F.R.C.S. Edin., M.R.C.P. Edin., Medical Superintendent. City Asylum, Hellesdon, Norwich.

1686. Harvey, Crosbie Bagenal, L.A.H., Assistant Medical Officer, District Asylum, Clonmel.

1892. Haslett, William John. M.R.C.S., L.R.C P., Resident Medical Suparintendent, Halliford House, Sunbury-on-Thames

1892. Hatchell, J., F.R.C.P.I., District Asylum, Maryborough, Ireland.

1891. Havelock, John G., M.B., C.M. Edin., Physician \&uperintendent, Montrose Royal Asvlum.

1890. Hay, Frank, M.B., C.M... Assistant Medical Officer, Ashburn Hall Asvlum, Dunedin, New Zealand.

1885. Henloy E. W., I.R.C.P., Courty Asylum, Gloucester.

1895. Hearder, Frederick P., M.B.. C.M., Assistant Medical Officer, West Riding Asylum. Wakefield.

1877. Hotherington, Charles, M.B., Medical Superintendent, District Asylum, Londonderry, Ireland.

1877. Hewson, R. W., L.R.C.P. Edin., Medical Supt., Coton Hill, Stafford.

1891. Heygate, Williạm Harris, M.R.C.S. Eng., L.S.A., Cranmere, Cosham, Hants.

1879. Hicks, Henry, M.D. St. And., M.R.C.S. Eng., F.R.S., F.G.S., Hendon Grove House, Hendon, Middlesex.

1882. Hill, Dr. H. Gardiner, Medical Superintendent, Middlesex County Asylum, Tooting.

1857. Hills, William Charles, M.D. Aber., M.R.C.S. Eng., Thorpe St. Andrew, near Norwich.

1871. Hingston. J. Tregelles, M.R.C.S. Eng., Medical Superintendent, North Riding Asylum, Clifton, York.

1881. Hitchcock, Charles Knight, M.D., Bootham Asylum, York.

1892. Holmes, James, M.D. Edin., Overdale Asylum. Whitefield. Lancashire.

1896. Horton. James Henry, M.R.C.S Eng., L.R.C.P. Lond., Assistant Medical Officer, The Priory, Roehampron, London, 8.W.

1896. Hossack, William Cardiff, M.B., C.M. Aberd., Assistant Physician, St. Catherine's. Banff.

1894. Hotchkiss, R. D., M.B., C.M., M.P.C., Assistant Physician, Royal Asylum. Glasgow.

1857. Humphry. J., M R.C.S. Eng., Medical Superintendent, County Asylum, Aylesbury, Bucks. 
1897. Hunter, David, M.A., M.B., B.C. Cantab., County Anylum, Whittingham.

1888. Hyslop, Theo. B., M.D., C.M. Edin., M.R.C.P.E., M.P.C., Assistant Medical Officer, Bethlem Royal Hospital, 8.E.

1882. Hyslop, James, M.D., Pietermariteburg Asylum, Natal, 8. Africa.

1865. Iles, Daniel, M.R.C.S. Eng., Resident Medical Officer, Fairford House

1871. Ireland, W. W., M.D. Edin., Mavisbush, Polton, Midlothian.

1896. Isacke, Mattherv W. S., M.R.C.S. Eng., L.R.C.P. Lond., Assistant Medical Officer, West Riding Asylum, Wadsley, Sheffield.

1866. Jacksun, J. Hughlings, M.D. St. And., F.R.C.P. Lond., Physician to the Hospital for Epilepsy and Paralysis, etc.; 3, Manchester Square, London, W.

1860. Jepson, Octavius, M.D. St. And., M.R.C.S. Eng., Elmfield, Ner.

1893. Johnston, Gerald Herbert, L.R.C.S. and L.R.C.P. Edin., Assistant Medical Officer, North Riding Asylum, Clifton, Yorks.

1890. Johnstou, .Tohn McCubbin, M.B., C.M., M.P.C., Town's Hospital, Parliamentary Road, Glasgow.

1878. Johnstone, J. Carlyle, M.D., C.M., Medical Superintendent, Roxburgh Disorict Asylum, Melrose.

1866. Jones, Evan, M.K.C.S. Eng., Ty-mawr, Aberdare, Glamorganshire.

1880. Jones, D. Johnson, M.D. Edin., Senior Assistant Medical Officer, Banstead Asylum, Surrey.

1882. Jones, Robert, M.D. Lond., B.S., F.R.C.S., Medical Superintendent, London County Asylum, Claybury, Woodford, Essex. (Gen. Seeretary.)

1897. Jones, Samuel Lloyd, M.R.C.S. Eng., L.R.C.P. Lond., Assist. Medical Officer, London County Asylum, Colney Hatch, N.

1897. Jones, William Fdward, Assistant Medical Officer, Earlswood Asylum, Redhill.

1879. Kay, Walter S., M.D., Medical Superintendent, South Yorkshire Asylum, Wadsley, near Sheffield.

1886. Keas, John, M B., Medical Superintendent, District Asylum, Inverness.

1894. Ker, Hugh Richard, F.R.C.S. Edin., M.R.C.S. Eng., L.R.C.P. Edin., Tintern, 2, Balham Hill, S.W.

1897. Kerr, Hugh, M.A., M.B., C.M, Assistant Medical Officer, Bucks County Asylum, Stone, Bucks.

1893. Kershaw, Herbert Warren, M.R.C.S. Eng., L.R.C.P. Lond., Senior

Assistant Medical Ofticer, North Riding Asylum, Clifton, Yorks.
1897. Kestezen, William Henry, M.R.C.S. Eng., L.S.A. Lond, Hillwood, Waverley Grove, Hendon.

1897. Kidd, Harold Andrew, M.R.C.S. Eng., L.R.C.P. Lond., Medicas Superintendert, West Sussex Asylum, Chichester.

1896. Langdon-Down, Reginald L., M.B., B.C. Cantab., M.R.C.P. Lond., Normansfield, Hampton Wick.

1896. Iaslett, Maurice H., L.R.C.P., Assistant Modical Officer, Somerset and Bath Asylum, Wells.

1892. Lawless, Dr. George Robert, A.M.O., District Asylum, Sligo.

1870. Iawrence, A., M.D., County Asylum, Chester.

1883. Iayton, Henry A. L.R.C.P. Edin., Cornwall County Asylum, Bodmin.

1883. Logge, R. J., M.D., Medical Superintendent, County Asylum, Derby.

1894. Iontagne, John, B.A., F.R.C.S.I., Medical Visitor of Lunatics to the Court of Chancery, 29, Westland Row, Dublin.

1858. Lowis, Henry, M D. Brux., M.R.C.S. Eng., L.S.A., late .Assistant Medical Officer, County Asylum, Chester ; West Terrace, Folkestone, Kent. 
1858. Lewis, Henry, M.D. Brux., M.R.C.S. Eng., L.S.A., lato Asvistant Riding Asylum, Wakefield.

1863. Loy, H. Rooke, M.R.C.S Eng., Medical Superintendent, County Anylum, Prestwich, near Manchester.

1859. Lindsay, James Murray, M.D. St. And., F R.C.S. and F.R.C.P. Edidin., Brookside, Corston, Bristol. (President, 1893.)

1883. Lisle, 8. Ernest de, I.R.C.P.I., Three Counties Asylums, Stotfold, Baldock

1872. Lyle, Thomas, M.D. Glasg., 34, Jesmond Road, Newcastle-on-Tyne.

1890. Lyons, Algernon Wilson, M.B. Lond., M.R.C.S., L.R.C.P., Thames Ditton, Burrey.

1880. MacBryan, Henry C., Kingsdown House, Box.

1897. McCutchan, William Arthur, L.R.C.P.S. Edin., Assistant Medical Officer, County and City Asylum, Heroford.

1884. Macdonald, P. W., M.D., C.M., Medical Superintendent, County Asylum, near Dorchester, Dorset. (Hon. Sec. S.W. Division.

1893. Macevov, Henry John, M.D., B.Sc. Lond., M.P.C., 41, Buckley Road, Brondesbury, London, N.W.

1895. Macfarlane, Neil M., M.D. Aberd., Medical Superintendent, Government Hospital, Thlotse Heights, Laribe. Basutoland, South Africe

1883. Macfarlane, W. H., M.B. and Ch.B. Univ. of Melbourne, Medical Supt., Hospital for the Insane, New Norfolk, Tasmania.

1891. Mackenzie, Henry J., M.B., C.M. Edin., M.P.C., Assistant Medical Officer, The Retreat, York.

1886. Mackenzie, J. Cumming, M.B., C.M., M.P.C., late Medical Superintendent. District Asylum, Inverness; care of Mr. Mackenzio, Enzio Station, Buckie, N.B.

- Mackintosh, Donald, M.D. Durh. and Glasg., L.F.P.S. Glasg., 10 Lancaster Road, Belsize Park, N.W.

1896. Maclaren. J., M.B., C.M. Edin., Aseistant Medical Officer, Spring Villa. Oughtybridge, Shofficld

1896. Maclean, A., M.D., J.P., St. Martin's Weymouth.

1886. Maclean, Allan, L.R.C.S. Edin., 10, Mitre Court Chambers, Temple, E.C.

1873. Macleod, M. D., M.B., Medical Superintendent, East Riding Asylum, Beverley, Yorks.

1882. Macphail. Dr. S. Rutherford, Derby Borough Asylum, Rowditch, Derby.

1896. Macpherson, Dr. Charles, Deputy Commissioner in Lunacy, 51, Queen Street, Edinburgh.

1895. Madge, Arthur E., M.R.C.S. Eng., L.R.C.P. Lond., Ivy House, St. Albans.

1896. Maguire, Charles Evan, M.B., C.M., Assistant Medical Officer, Durham County Asylum. Winterton, Ferryhill.

1896. Mallanah, S., M.B. Edin., Medical School, Hyderabad, Deccan, India.

1865. Manning, Harry, B.A. Lond., M.R.C.S., Laverstock House Salisbury.

1896. Marr, Hamilton C., M.D. Glasg. Univ., Senior Assistant Physician, Wcodileo Asylum, Lenrio.

1897. Marshall, John, M.B., C.M. Glasg., Assistant Medical Officer, County Asylum. Bridgend, Glamorgan.

- Marshall, William G., F.R.C.S., 72, Bromfelde Road, Clapham, S.W.

1896. Martin Janies Clarke, L.R.C.S.I., I.M. I.R.C.P., Aexistant Medical Officer, District Asylum, Letterkenny.

1897. Mathicson, M. B., C.M. Glasg., Senior Assistant Medical Officer, County Asylum. Stafford.

1888. McAlister, William, M.B., C.M., Struen Villas, Kilmarnock.

1894. McClaughry, Thomas, L.R.C.S.I. and L.A.H. Dubl., Assistant Medical Officer, District Asylum, Maryborough, Ireland. 
1886. McCreery, James Vernon, L.R.C.S.I., Medical Superintendent, Now Lunatic Asylum, Melbourne, Australia.

1870. McDowall, T. W., M.D. Edin., L.R.C.S.E., Medical Superintendent, Northumberland County Asylum, Morpeth. (Presidintr.)

1876. McDowall, John Greig, M.B. Edin., Medical Superintendent, West Riding Asylum, Menston, near Loeds.

1882. McNaughton, John, M.D., Medical Superintendent, Criminal Lunatio Asylum, Perth.

1894. McWilliam, Alexander, M.B. Aberd., Senior Assistant Medical Officer, Heigham Hall, Norwich.

1886. Macpherson, John, M.B., M.P.C., Medical Superintendent, Stirling Asylum. Larbert.

1890. Mentiass, W. F., M.D., B.Sc. Edin., Senior Assistant Medical Officer, County Asylum, Rainhill.

1891. Mercier, Charles A., M.B. Lond., F.R.C.S. Eng., Lecturer on Insanity, Westminster Hospital ; Flower House, Catford, S.E

1877. Marson, John, M.D. Aberd., Medical Superintendent Borough Asylum, Hull.

1871. Mickle, Willium Julius, M.D., F.R.C.P. Lond., Medical Superintenrent, Grove Hall Asylum, Bow, London. (Ex-Prestdentr.)

1867. Mickley, George, M.A., M.B. Cantab., Medical Superintendent, St. Luke's Hospital, Old Street, London, E.C.

1893. Middlemass, James, M.B., C.M., B.Sc. Edin., Senior Assistant Physician, Royal Edinburgh Asylum.

1883. Miles, George E., M.R.C.P., etc., Medical Superintendent, Hospital for the Insans (Idiots), Newcastle, N.S.W.

1897. Millard, Reginald J., M.B., Ch.M., Sydney, Assistant Medical Officer, Callan Park, Sydney, N.S.W.

1893. Mills, John, M.B., B.Ch., and Diploma in Mental Diseases, Royal University of Ireland, Assistant Medical Officer, District Asylum. Ballinasloe.

1887. Miller, Alfred, M.B. and B.C. Dubl., Medical Superintendent, Hatton Asylum, Warwick.

1881. Mitchell, R. B., M.D., Medical Supt., Midlothian District Asvlum.

1895. Moffett, Elizabeth Jane, M.B., B.Sc. Lond, Junior Assistant Medical Officar, District Asylum, Mullingar.

1885. Molony, John, F.R.C.P.I., Mod. Supt., St. Patrick's Hospital, Dublin.

1897. Montgomery, Sydney Hamilton Rowan, M.B., B.Ch., B.A.O. Royal Univ. Irel., Assistant Medical Officer, Borough Asylum, Nottingham.

1878. Moodv, James M., M.R.C.S. Eng., 'L.R.C.P. and L.M. Edin., Medical Superintendent, County Asylum, Cane Hill, Surrey.

1885. Moore, E. E., M.B. Dubl., M.P.C., Medical Superintendent, District Asplum, Letterkennv, Ireland

1891. Moore, George, J.P., M.D., M.R.C.S., Queen's Farm, St. Saviour's,

1897. Mornement, Robert Harry, M.R.C.S. Eng., L.R.C.P. Lond., Assistant Medical Otficer, Iondon County Asylum, Cane Hill, Purley, Surrev.

1892. Morrison, Cuthbert S., L.R.C.P. and L.R.C.S. Edin., Medical Superintendent, County and City Asylum. Burghill, Hereford.

1896. Morton, W. B., M.B., Assistant Medical Officer, Brislington House, Bristol.

1896. Mott. F. W.. M.D., B.S., F.R.C.P. Lond., F R.S., 84, Wimpole Street. W.; Pathologist, Iondon County Asylum; Assistant Phvsician, Charing Cross Hospital

1896. Mould, G.E., M.R.C.S., L.R.C.P. Lond., Medical Superintendent, Northumberland House. Finsbury Park, London, N.

1862. Mould, George W., M.R.C.S. Eng., Medical Superintendent, Royal Lunatio Hoopital, Cheadlo, Manchester. (Prasmenrs, 1880.) 
1897. Mould, Philip G., M.R.C.S. Eng., L.R.C.P. Lond., Assistant Medical

1878. Muirhead, Claud, M.D., F.R.C.P. Edin., 30, Charlotte Square, Edinburgh.

1897. Mumbr, Bonner Harris, M.D. Aberd., D.P.H. Cantab., Medical Suparintendent, Borough Asylum, Portsmouth.

1893. Murdoch, James William Aitken, M.B., C.M. Glasg., Medical Buperintendent, Berks County Asylum, Wallingford.

1878. Murray, Henry G., L.R.C.P. Irel., L.M., L.R.C.S.I., Assistant Medical Officer, Prestwich Asylum, Manchester.

1891. Muggrove, C. D., Dr., 8, Herbert Terrace, Penarth, S. Wales.

1890. Nash, Vincent, L.R.C.P.I., formerly Assistant Medical Officer, Richmond District Asylum, Dublin; George's Street, Limerick.

1880. Neil, James, M.D., M.P.C., Assistant Medical Officer, Warnoford Asylum, Oxford.

1875. Nowingtion, Alexander, M.B. Camb., M.R.C.S. Eng., Woodlands,

1873. Newington, H. Hayes, M.R.C.P. Edin., M R.C.S. Eng., Ticehurst, Sussex. (Presiuner, 1889.) (Treasurer.)

1893. Newington, John, L.S.A., Tattlebury House, Goudhurst, Kent.

1881. Nowth, A. H., M.D., Haywards Heath, Sussex.

1869. Nicolson, David, M.D. and C.M. Aberd., late Medical Officer, H.M. Convict Prison, Portsmouth, and State Asylum, Broadmoor; Lord Chancellor's Visitor, Elmhurst, Guildford. (Presidenst, 1895.)

1895. Nicolson, Robert Henderson, M.B., C.M. Aberd, Senior Assistant Melical Officer, Ccunty Asylum, Hatton, Warwick.

1893. Nobbs, Athelstane, M.B., C.M. Edin., 339, Queen's Road, Battersea Park, S.W.

1888. Nolan, Michaol J., L.R.C.P.I., M.P.C., Medical Suporintendent, District Asylum, Downpatrick.

1892. Noott, Reginald Harry, M.B.. C.M. Edin., Senior Assistant Medical Officer, Broadmuor Criminal Lunatic Asylum, Crowthorne, Wokingham.

1880. Norman, Conolly, F.R.C.P.I., Medical Superintendent, Richmond District Asylum, Dublin, Ireland. (Hon. Secretary for Ireland, 1837-94.) (PrEsident, 1895.) (Editor of Journal.)

1885. Oakshott, J. A., M.D., Assist. Medical Officer, District Asylum, Cork.

1892. O'Mara, Dr., District Asylum, Limerick, Ireland.

1881. O'Meara, T. P., M.B., Med. Supt, District Asylum, Carlow, Ireland.

1885. O'Neill, F. D., L.R.C.P.I., Medical Supt., The Asylum, Limerick

1897. Orange. Margaret, L.S.A. Lond., M.B. Brux., Assistant Medical

Officr. London County Asylum, Claybury, Essex.

1868. Orange, William, M.D. Heidelb., F.R.C.P. Lond., C.B., The Bryn, Godalming, Surrey. (President, 1883.)

1893. Osburne, Cecil A. P., F.R.C.S. Edin., L.R.C.P. Edin., Surgeon to the Admiralty, The Grove, Catton, Norwich.

1890. Oswald, Landel R., M.B., M.P.C., Medical Superintendent, City of Glasgow District Asylum, Gartcash, N.B.

- Palmer, Edwand, M.D. St. And., M.R.C.P. Lond., M.R.C.8., 87, Harcourt Terrace, London, S.W.

1893. Paterson, Charles Edward, M.D. Edin., Arnold House, Farnborough, Hents.

1892. Patterson, Arthur Edward, M.B., C.M. Aberd., Assistant Medical Officer, City cf London Asylum, Dartford. 
1872. Patton, Alex., M.B., Resident Medical Superintendent, Farnham House, Finglas, Co. Dublin.

- Paul, John Hayball., M.D. St. And., M.R.C.P. Lond., F.R.C.P. Edin., Camberwell Terrace, London, S.E. (Emerilus Treasurer.)

1889. Peacock, Dr., L.R.C.P. and L.M. Edin., M.R.C.S. and L.S.A. Lond., Resident Medical Officer and Proprietor, Ashwood House, Kingswinford, Dudley, Staffordshire.

1673. Pedler, George H., L.R.C.P. Lond., M.R.C.S. Eng., 6, Trevor Terrace, Knightsbridge, S.W.

1893. Perceval, Hrank, M.R.C.S. Eng., L.R.C.P. Lond., Medical Superintendent, County Asvlum, Whittingham, Preston, Lancashire.

1874. Petit, Joseph, L.R.C.S.I., Medical Supt., District Asylum, Sligo.

1878. Philipps, Sutherland Rees, M.D., C.M. Queen's Univ. Irel., F.R.G.S., St. Anne's Heath, Chertsey.

1875. Philipson, George Hare, M.D. and M.A. Cantab, F.R.C.P. Lond., 7, Eldon Square, Newcastle-on-Tyne.

1891. Pierce, Bedford, M.D. Lond., M.R.C.P., Medical Superintendent, The Retreat, York.

1538. Pieter32n, J. F. G., M.R.C.S., Ashwood House, Kingswinford, neax Dudley, Stafford.

1871. Pim, F. Esq., M.R.C.S. Eng., L.R.C.P. Irel., Medical Superintendent, Palmerston, Chapelizod, Co. Dublin, Ireland.

1890. Pitcairn, J. J., L.R.C.P., M.R.C.S., M.P.C., 1, Parkhurst Road, Holloway, $\mathrm{N}$.

1896. Planck, Charles, M.R.C.S. Eng., L.R.C.P. Lond., M.A. Camb. Assistant Medical Cifficer, East Sussex County Asylum, Haywards Heath.

1877. Plaxton, Joseph William, M.R.C.S., L.S.A. Eng., Lunatic Asylum, Kingston, Jamaica.

1889. Pope, George Stevens, L.R.C.P. and L.R.C.S. Edin., I.F.P. and S. Glasg. Medical Superintendent, Middlesborough Asylum, Cleveland, Yorks.

1876 Powell, Evan, M.R.C.S. Eng., L.S.A., Medical Superinterdent, Bcrough Lunatic Asylum, Nottingham.

1891. Price, Arthur, M.R.C.S., L.S.A., M.P.C., Merriebank, Moss Lano, Aintree, Liverpool.

1675. Pringie, H. T., M.D. Glasg., Medical Superintendent, County Asylum, Bridgend, Glamorgan.

1894. Rambant, Daniel F.. M.D. Univ. Dubl., Third Aesistant Medical Officer, and Pathologist, Richmond District Asylum, Dublin.

1889. Raw, Nathan, M.D., M.P.C., Roval Infirmary, Dundee.

1893. Rawes, William, M.B. Durh., F.R.C.S. Eng., Assistant Medical Officer, St. Luke's Hospital, London.

1896. Ray, Matthew B., M.B., C.M. Edin., Pathologist and Assistant Medical Officer, West Riding Asylum, Wadsley, Sheffield.

:870. Rayner, Henry, M.D. Aberd., M.R.C.P. Edin , 2, Harley Street, london, W., and Upper Terrace House, Hampstead, London, N.W. (President, 1884.) (Late General Secretary.) (Editor of Journal.)

1887. Roid, William, M.D., Physician Superintendent, Royal Asylum, Absrdeen.

1891. Renton, Robert, M.B., C.M. Edin., M.P.C., Montague Lawn, London Road, Cheltenham

1897. Renton, James Murray, M.B., C.M. Edin., Assistant Medical Officer, Colinty Asylum, Chester.

1886. Revington, George, M.D. and Stewart Scholar Univ. Dubl., M.P.C. Medical Supt. Contral Criminal Asylum. Dundrum, Ireland.

1897. Richard, William J., M.A., M.B., C.M. Glasg., Medical Officer, Govan Parochial Asylum, Merryflats, Govan. 
1889. Richards Joseph Peeke, M.R.C.S., L.S.A , 6, Freeland Road, Ealing, W.

1893. Rivers, William H. Rirers, M.D. Lcnd., St. John's College, Cambritge.

1871. Robertson, Alexander, M.D. Edin., 16, Newton Terrace, Glasgow.

1895. Robertson, William Ford, M.B., O.M., Pathologist, Royal Edinburgh Asylum, West House, Morningsido Asplum. Edinburgh.

1887. Robertson, G. M., M.B., C.M., M.P.C., Medical Superintendent, Perth District Asylum, Murthley.

1895. Robinsion, George Burton, M.B., L.R.C.P., M.R.C.S., County Asylum, Morpeth.

1876. Rogers, Edward Coulton, M.R.C.S. Eng., L.S.A., County Asylum, Fulbourn, Cambridge.

1859. Rogers, Thomas Lawes, M.D. St. And., M.R.C.P. Lond., M.R.C.S. Eng., Eastbank, Court Road, Eltham, Kent. (President, 1874.)

1895. Rolleston, Iancelot W., M.B., B.S. Durh., Senior Assistant Medical Officer, Middlesex County Asylum, Tooting, S.W.

1879. Ronældson, J. B., L.R.C.P. Edin., Medical Officer, District Asylum, Haddington.

1879. Roots, William H., M.R.C.S., Canbury House, Kingston-on-Thames.

1860. Rorie, James, M.D. Edin., L.R.C.S. Edin., Medical Superintendent, Roval Asylum, Dundee. (Late Hon. Sccretary for Scotland.)

1888. Ross, Chisholm, M.B. Edin., M.D. Sydney, Hospital for the Insane, Kenmore, New South Wales.

1884. Rowe, E. L., L.R.C.P., Edin., Med. Supt., Borough Asylum, Ipswich.

1883. Rowland, E. D., M.D., C.M. Edin., The Public Lunatic Asylum, Berbice, British Guiana.

1877. Russell, A. P., M.B. Edin., The Lawn, Lincoln.

1883. Russell, F. J.' R., L.R.C.P. Irel.

1892. Ruttiedge, Victor, M.B., District Asylum, Londonderry, Ireland.

- 1866. Rutherford, James, M.D. Edin., F.R.C.P. Edin., F.F.P.S. Glasgow, Physician Superintendent, Crichton Royal Institution, Dumfries. (Hon. Secretary for Scotland, 1876-86.)

1896. Rutherford, James M., M.B., C.M. Edin., Assistant Physician, Royal Edinburgh Asvlum, Morningside.

1887. Rutherford, W., M.D., Consulting Physician, Ballinasloe District Asylum, Ireland.

1896. Rutherford, Robert Leonard, M.D., Medical Superintendent, Digby's Asylum, Exeter.

1889. Ruxton, William Leddington, M.D. and C.M., 8, Derwent Place, Newcastle-on-Tyne.

- Sunkey, R. Heurtley H., M.R.C.S. Eng., Modical Superintendent, Oxford County Asylum, Littlemore, Oxford.

1894. Sankey, Edward H. O., M.A., M.B., B.C. Cantab., Resident Medical Licensee, Boreatton Park Licensed House, Baschurch, Salop.

1891. Saundars, Charles Edwards, M.D. Aberd., M.R.C.P. Lond., Medical Superintendent, Haywards Heath Asylum, Sussex.

1873. Savage, G. H., M.D. Lond., 3, Henrietta Street, Cavendish Square. W. (Late Editor of Journal.) (PREsIDENT. 1886.)

1894. Scanlan, Willizm T. A., M.B., M.Ch., B.A.O.R.U.I., Assistant Medical Officer, District Asylum, Cork.

1862. Schofield, Frank, M.D. St. And., M.R.C.S., Medical Superintendent Camberwell House, Camberwell

1884. Scott, J. Walter, M.R.C.S., M.P.C., Highfield, Tulse Hill, S.W.

1896. Scott, James, M.B., C.M. Edin., Medical Officer, H.M. Prisons, Holloway and Newgate; 3, Parkhurst Road, Holloway, London, N.

1889. Scowcroft, Walter, M.R.C.S , Senior Assistant Medical Officer, Royal Lunstic Hospital, Cheadle

1880. Soccombe, George: L.R.C.P.L., The Colonial Lunatic Asylum, Port of Spain, Trinidad, West Indies. 
1879. Seed, William, M.B., C M. Edin., The Poplars, 110, Watorloo Road, Ashton-on-Ribble, Preston.

1889. Sells, Charles John, L.R.C.P., M.R.C.S., L.S.A., White Hall, Guildford.

1882. Seward, W. J., M.D., Medical Supt., Colney Hatch, Middlesex.

1896. Shanahan, John Francis, L.R.C.P.I., L.R.C.S.I., 2, The Crescent, Jimerick.

1891. Shaw, John Custance, M.R.C.S. Eng., L.R.C.P. Lond., 4, Outer Temple, Strand, London.

1867. Shaw, Thomas C., M.D. Iond., F.R.C.P. Lond., Medical Superintendent, London County Asylum, Banstead, Surrey.

1820. Shaw, James, M.D., 310, Kensington, Liverpool.

1891. Shaw, Harold B., B.A., M.B., B.B., D.P.H. Camb., Medical Supt., Isle of Wight County Asylum, Whitecroft, Newport, I. of W.

1882. Sheldon, T. S., M.B., Medical Superintendent, Cheshire County Asylum, Parkside. Macclesfield.

1886. Sherrara, C. D., M.R.C.S., Avalon, Eastbourne

1896. Shortt, William Rushton, M.B., B.S. Durh., M.R.C.S., L.R.C.P. Lond., Assistant Medical Officer, City Asylum, Gosforth, Newcastle-on-Tyne.

1877. Shuttlew orth, G. E., M.D. Heidelb., M.R.C.S. and L.S.A. Eng., B.A. Lond., late Medical Superintendent, Royal Albert Asylum, Lancaster; Ancaster House, Richmond Hill, Surrey.

1895. Simpson, Francis, M R.C.S., I.R.C.P., Assistant Medical Officar, West Riding Asylum, Wakefield.

1889. Simpson, Samuel, M.B. and M.C.H. Dubl., M.P.C., Balieboro', Co. Cavan, Ireland.

1888. Sinclair, Eric, M.D., Medical Suporintendent, Gladesville Asylum, New South Wales.

1870. Skae, C. H., M.D. St. And., Medical Superintendent, Ayrshire District Asylum, Glengall, Arr.

1891. Skeen, James Humphrey, M.B., C.M. Aberd., Medical Superintendent, Glasgow District Asylum, Bothwell.

1858. Smith, Robert, M.D. Aberd., L.R.C.S. Edin., Medical Superintendent, County Asylum. Sedgefield, Durham.

1886. Smith, R. Gillies, M.A., B.Sc., M.R.C.S., City Asylum, Gosforth, Newcastle-on-Tyne.

1885. Smith. R. Percy, M.D.. B S., F.R.C.P., M.P.C., Bethlem Hospital, St. George's Road, S.E. (General Secretary, 1896-7.)

1884. Smith, W. Beattie, F.R.C.S. Edin., I.R.C.P. Lond., Medical Superintendent, Hospital for the Insans, Ararat, Victoria.

i892. Smyth, W. Johnson, M.B. Edin., Guards' Hospital, London.

1881. Snell, George, M.D. Aberd., M.R.C.S. Eng., Medical Superintendent, Public Lunatic Asylum, Berbice, British Guiana.

1885. Soutar, J. G., Barnwood House, Gloucester.

1875. Spence, J. Beveridge, M.D., M.C. Queen's Univ., Medical Superintendent, Burntwood Asylum, near Lichfield. (Registrar.)

1883. Spence, J. B., M.D., M.C., care of Alex. Philip, Esq., 16, Panmure Street, Brechin.

18:1. Stansfield, T. E. K., M.B., C.M. Edin., Senior Assistant Medical Officer, London County Asylum, Claybury.

1895. Stanwell, Charles Oliver, L.R.C.P. and S. and L.M. Edin., Senior Assistant Medical Officer, The Retreat, York.

1868. Stewart, James, B.A. Queen's Univ., F.R.C.P. Edin., I.R.C.S. Irel., late Assistant Modical Officer, Bent County Asylum, Maidstone; Dunmurry, Sneyd Park, near Clifton, Gloucestershire.

1884. Stewart. Robert S., M D., C.M., Assistant Medical Officer, County Asyluin, Glamorgan.

1887. Stowart, Rothsay C.. M.R.C.S., Assistant Medical Officer, County Asylum, Leicester. 
1862. Stilwell, Henry, M.D. Edin., M.R.C.S. Eng.: Moorcroft House, Hillingdon, Middlesex.

1864. Stocker, Alonzo Henry, M.D. St. And., M.R.C.P. Lond., M.R.C.S. Eng., Medical Supt., Peckham House Asvlum, Peckham.

1897. Stoddart, William Henry Butler, M.B., B.S. Lond., M.R.C.S. Eng., L.R.C.P. Lond., Clinical Assistant, Rethlem Royal Hospital, S.E.

1881. Strahan, S. A. K., M.D., Assistant Medical Officer, County Asylum, Be:rywood, near Northamption.

1868. Strange, Arthur, M.D. Edin., Medical Superintendent, Salop and Montgomery Asylum, Bicton, near Shrewsbury.

1895. Strapp, Walter Russell, M.B., C.M., care of Mrs. Warrington, Aberfoyle.

1896. Strzion, Charles Robert, F.R.C.S. Edin., Medical Visitor, Fisherton House and Laverstock House, West Lodge, Wilton, Wilts.

1885. Street, C. T., M.R.C.S., L.R.C.P., Haydock Lodge, Ashton, Newtonb-Willows, Lancashire.

1386. Suffern, A. C., M.D., Medical Superintendent, Rubery Hill Asylum, near Bromsgrove, Worcestershire

1894. Sullivan, W. C., M.D.R.U.I., 74, Oakley Street, Chelsea, London, S.w.

1870. Sutherland, Henry, M.D. Oxon., M.R.C.P. Lond., 21, New Carendish Street, Portland Place, W.; Newlands House, Tooting Bec Road, Tooting Common, S.W. ; and Otto House, 47, Northend Road, West Kensington, W.

1895. Sutherland, John Francis, M.D. Edin., Deputy Commissioner in Lunacy, 4, Merchiston Bank Avenue, Edinburgh.

1E68. Swain, Edward, M.R.C.S., Medical Superintendent, Three Counties' Asylum, near Hitchin, Herts.

1877. Swanson, George J., M.D. Edin., Lawrence House, York.

1897. Tait, James Siaclair, M.D., L.R.C.P. Lond., L.R.C.S. Edin., Medical Superintendent, Hospital for Insane, St. Johns, Newfoundland.

18ET. Tate, Wiliiam Barney, M.D. Aberd., M.R.C.P. Lond,, M.R.C.S. Eng., Med. Supt. of the Lunatic Hospital, The Coppice, Nottingham.

1897. Taylor, Frederic Ryott Perciral, M.D., B.S. Lond., M.R.C.S. Eng., L.R.C.P. Lond., Assist int Medical Officer, London County Asylum, Claybury.

1890. Telford-Smith, 'Telford, M.A., M.D., Medical Superintendent, Royal Albert Asylum, Lancaster.

1888. Thomas, E. G., Park House, Caterham, Surrey.

1880. Thomson, D. G., M.D., C.M., Medical Superintendent, County Asylum, Thorpe, Norfolk.

1897. Thurman, William Rowland, M.B., B.S. Dunedin, Assistant Medical Officer, City and County Asylum, Bristol.

1896. Townsend, Arthur, M.R.C.S. Eng., I..R.C.P. Lond., Assistant Medical Officer, Hospital for Insane, Barnwood House, Gloucester.

1866. Tuke, John Batty, M.D. Edin., 20, Charlotte Square, Edinburgh. (Hon. Secretary for Scotland, 1869-72.)

1888. Tuke, John Ba.tty, junior, M.B., C.M., M.R.C.P.E., Resident Physician, Saughton Hall, Edinburgh.

1881. Tuke, Charles Molesworth, M.R.C.S.E., Chiswick House, Chiswick.

1885. Tuke, T. Soymour, M.B., B.Ch. Oxford, M.R.C.S.E., Chiswick House, Chiswick ; and 37, Albemarle Street, Piccadilly, W.

1877. Turnbull, Adam Robert, M.B., C.M. Edin., Medical Superintendent, Fife and Kinross District Asylum, Cupar. (Hon. Secretary for Scotland.)

1896. 'Turner, Alan Charles, M.R.C.S. Eng., L.R.C.P. Lond., 79, Gordon

1889. Turner, Alfred, M.D. and C.M., Assistant Medical Officer, West Riding Asylum, Menston, Yorkshire. 
1890. Turner, John, M.B., C.M. Aberd., Senior Asxistant Medical Officer, Essex County Asylum.

1878. Urquinart, Alexr. Reid, M.D., F.R.C.P.E., Physician Superintendent, James Murray's Royal Asylum, Perth. (Editor of Journal.) (Hon. Sceretary for Scotland, 1836-94.)

1694. Vincent, William James, M.B. Durh., Assistant Mecical Officar, Borough Asylum, Nottingham.

1876. Wade, Arthur Law, B.A., M.D. Dubl., Medical Superintendent, County Asylum, Wells, Somerset.

1884. Walker, E. B. C., M.B., C.M. Edin., Assistant Medical Officer, County Asylum, Haywards Heath.

1896. Walker, William F., L.R.C.S. and L.M. Edin., L.S.A. Iond., Co Proprietor and Licensee, Home for Inebriates, Strest Court, Kingsland, R.S.O., Herefordshire.

1877. Wallace, James, M.D., Visiting Medical Officer, 16, Union Street, Greenock.

1876. Wallis, John A., M.D. Aberd., L.R.C.P. Edin., Commissioner in lunacy, 19, Whitehall Place, S.W.

1883. Walmsley, F. H., M.D., Medical Superintendent, Darenth Asylum, Dartford, Kent.

1671. Ward, J. Bywater, B.A., M.D. Cantab., M.R.C.S. Eng., Medical Superintendent, Warneford Asylum, Oxford.

1889. Warnock, John, M.D., C.M., B.Sc., M.R.C.S., Sanitary Department, Ministry of Interior, Cairo, Egypt.

1897. Warren, Ernest Downing, I.R.C.P. Lond., M.R.C.S. Eng., Assistant Medical Officer, City Asylum, Digby's, Exeter.

1895. Waterson, Jane F.lizabeth, M.D. Brussels, L.R.C.P.I., L.R.C.S. Edin., Official Visitor, Cape Town District Lunatic Asylums, Cape Town, South Afriea.

1891. Watson, George A., M.B., C.M. Edin., M.P.C., Senior Assistant Medical Officer, City Asylum, Birmingham.

1885. Watson, William Riddell, L.R.C.S. and L.R.C.P. Edin., Govan District Asylum, Hawkhead, Paisley.

1897. Watt. Neish Park, M.B., C.M. Edin.

1880. Weatherly, Lionel A., M.D., Bailbrook House, Bath.

1897. Welsh, Gilbert Aitken, M.B., C.M. Edin., Assistant Physician, Crichton Royal Institution, Dumfries.

1880. West, George Francis, I.R.C.P. Edin., Assistant Medical Officer, District Asylum, Omagh, Ireland.

1672. Whitcombe, Edmund Banks. M.R.C.S., Medical Superintendent, Winson (Treen Asylum, Birmingham. (Presidest, 1891.)

1884. White, Ernest, M.B. Lond., M.R.C.P., City of Jondon Asvlum, Stone,

Dartiord, Kent.
1889. Whitwell, James Richard, M.D. and C.M., Medical Superintendent, Suffolk Countv Asylum, Melton Woodbridge.

1883. Wiglesworth, J., M.D. Lond. Rainhill Asylum, Lancrshire.

1895. Wilcox, Arthur William, M.B., C.M. Edin., Second Assistant Medical Officer, County Asylum, Hatton. Warwick.

1887. Will. John Kennely, M.B., C.M., M.P.C., Bethnal House, Cambridge Road. E.

1862. Williams, S. W. Duckworth, M.D. St. And., L.R.C.P. Lond., 76, Jermyn Street, London, S.W.

1890. Wilson. George R., M.B., C.M., M.P.C., Medical Superintendent, Mavisbank Asvlum, Polton, Midlothian.

1896. Wilson, Robert, M.B., C.M. Glasg., Nailsworth, Gloucestershire.

1895. Wilson, James, M.A., M.B., C.M., Assistant Medical Officer, Wilts County Asylum, Devizes. 
1875. Winslow, Henry Forbes, M.D. Lond., M.R.C.P. Lond., 14, York Place, Portman Square, Iondon, and Hayes Park, Hayes, near Uxbridge, Middlesex.

i897. Wissman, David William, M.R.C.S. Eng., L.R.C.P. Lond., Assistant Medical Cfticer, Countv Asvlum, Melton, Suffolk.

1869. Wood, T. Outterson, M.D., M.R.C.P. Lond., F.R.C.P., F.R.C.S. Edin., 40, Margaret Street, Cavendish Square, W.

1894. Wood, Guy Mills, M.B. Durh., Assistant Medical Officer, County Asyluin, Rainhill, near Prescot, Lancashire.

1873. Woods, Oscar T.. M.B., M.D. Dubl., L.R.C.S.I., Medical Superintendent, District Asylum, Cork. (Hon. Secretary for Ireland.)

1885. Woods, J. F., M.R.C.S., Medical Superintendent, Huxton House, N.

187. Wo:thington, 'Thomas Blair, M.A. M.B., and M.C. 'Trin. Coll. Dubl., Medical Supt., County Asylum, Knowle, Fareham, Hants.

1862. Yelluwlees, David, M.D. Edin., F.F.P.S. Glasg., LI.1)., Physician Superintendent, Royal Asylum, Gartnavel, Glasgow. ('PRrsIDENT, 1890.)

1882. Young, W. M., M.D., Steeven's Hospital, Dublin.

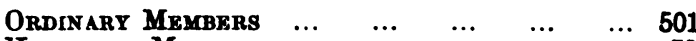

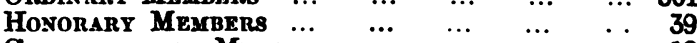

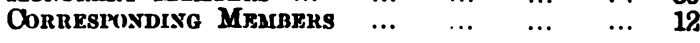

$$
\begin{aligned}
& \text { Total } \ldots \quad \ldots \quad \ldots \quad \ldots \overline{552}
\end{aligned}
$$

Members are particularly requested to send changes of address, etc., to Dr. Robert Jones, the Honorary Secretary, 11. Chandos Street, Cavendish Square, London, $W$., and in duplicate to the Printers of the Journal, South Counties Press Limited, Lewes, Swssex. 


\section{xxii}

List of those who have passed the Examination for the Certificate of Efficienoy in Psyohological Medicine, entitling them to append M.P.O. (Med. Psych Certif.) to their names.

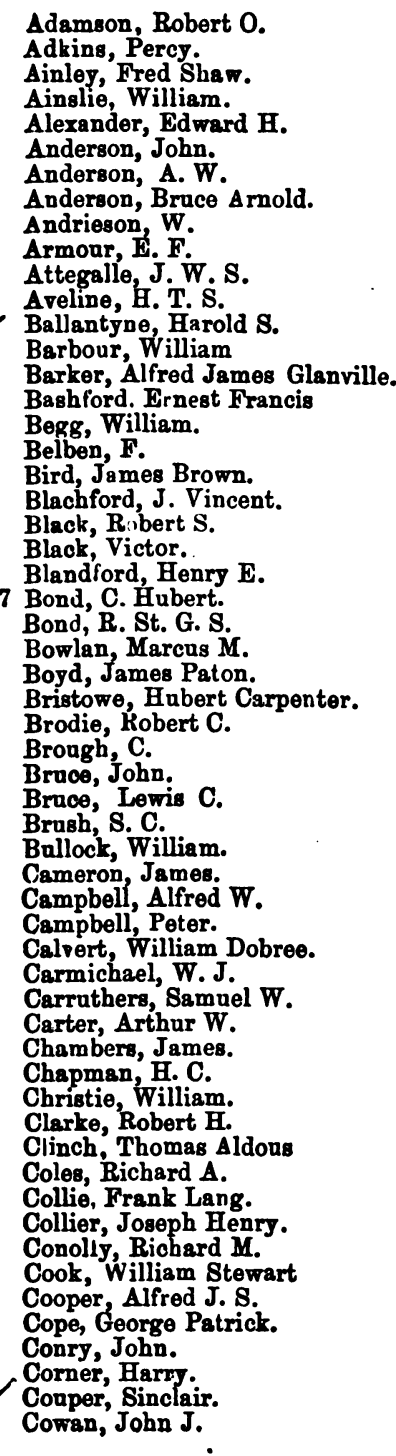

Cowie, C. G.

Cowie, George.

Cowper, John.

Craig, M.

Cram, John.

Cross, Edward John.

Cruickshank, Georgo.

Cullen, George M.

Dalgetty, Arihur B.

6 Dawson, W. R.

Davidson, William.

Davidson, Andrew.

De Silva, W. H.

Distin, Howard.

Druminond, Russell J.

Donald, Wm. D. M.

Donaldson, R. I. S.

Donellan, James O'Conor.

Douglas, A. R.

Eames, Henry Martyn.

Eames, Henry Mar, James H.

Eden, Richard A, S.

Edgerley, $\mathbf{S}$.

Edwards, Alez. H.

Elkins, Frank A.

Ellis, Clarence J.

English, Edgar.

Engtace, J. N.

Eustace, Henry Marcus.

Evans, P. C.

Ewan, John A.

Ezard, Ed. W.

Farquharson, Wm. Fredk.

Fennings, A. A.

Ferguson, Robert

Fitsgerald, Gerald

Fraser, Thomas.

Fraser, Donald Allan.

Frederick, Herbert John

Fox, F. G. T.

Gawn, frnest K.

Gemmell, William.

Genney, Fred. S.

Giles, A. B.

Gill, J. Macdonald.

Gilmour, John R.

Goldie, $\mathbf{B}$. M.

Goodall, Edwin.

Graham, F. B.

Graham, Dd. James.

Grainger, Thomas.

Grant,-J. Wemyss.

Grant, J. Weklan.

Gray, Alex. C. H.

Griffiths, Edward H. 


\section{Ixiii}

\begin{tabular}{|c|c|}
\hline 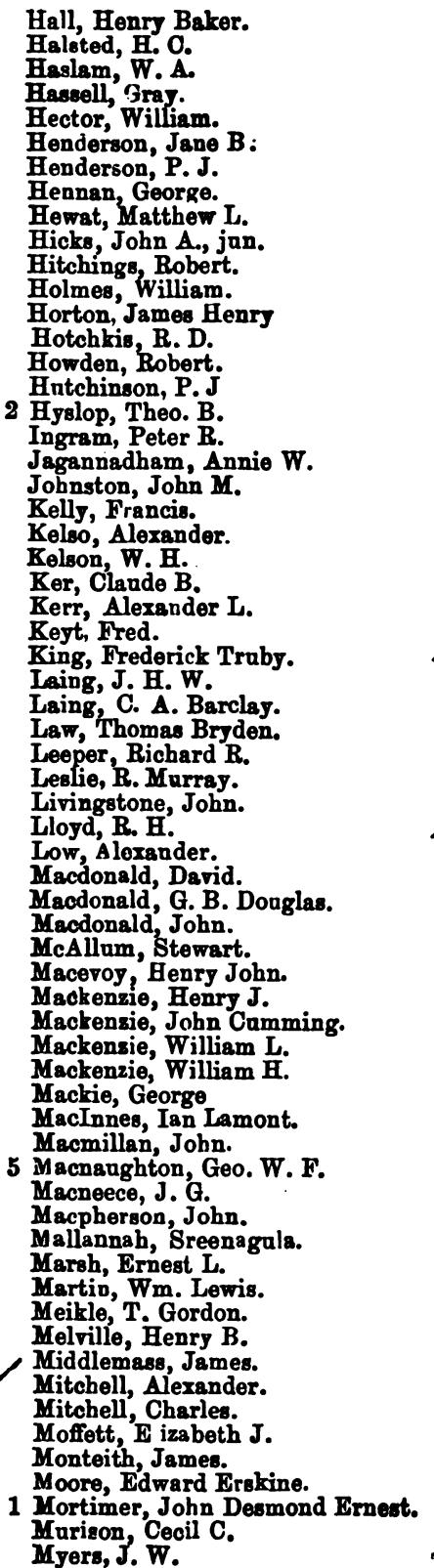 & 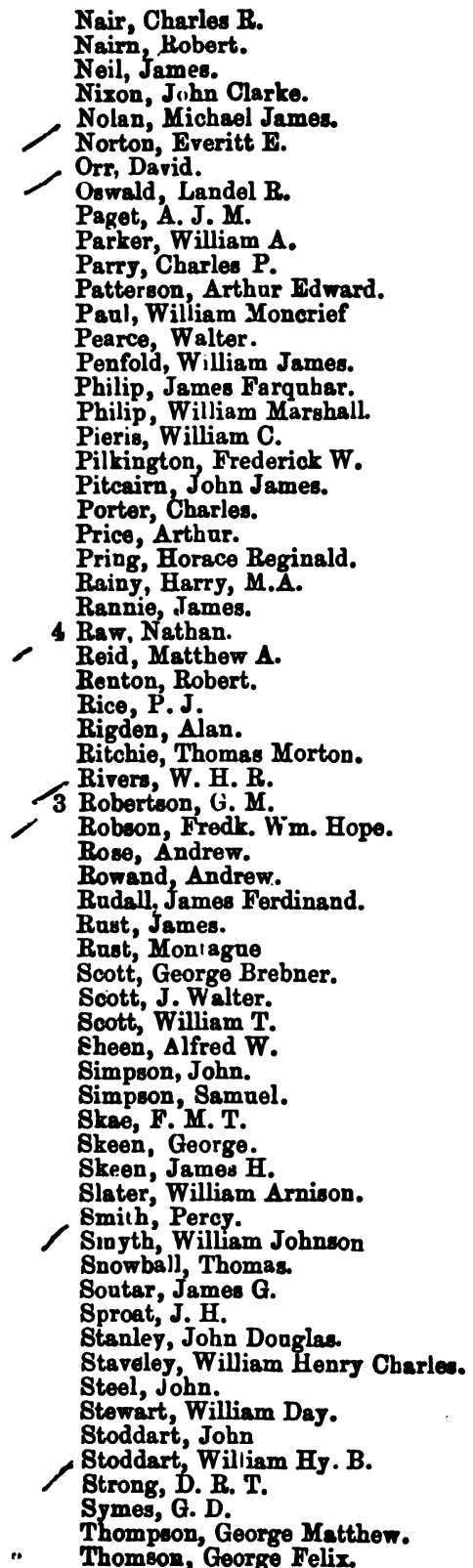 \\
\hline
\end{tabular}


sxiv

Thorpe, Arnold B.

Trotter, Robert Bamud.

Turner, W. A.

Umney, W. $\mathrm{T}$.

Walker, Jame.

Watereton, Jane Ilizabeth.

Wateon, George A.

Welnh, David 4 .

Weinh, J.T.

Wicham, Gilbert Fenry.

Whitwell, Robert R. H.

Will, JohnKennody.

Williame, D. J.

Williamson, 4 . Marwoll.

Wilson, John T.

Wileon, G. B.

Wilson, Jamen.

Wilson, Bobert.

Wood, Darid Jamea.

Yeoman, John B.

Yeates, Thoman.

Young. D. P.

Younger, Henry J.

Zimmex, Carlo Raymond.

1 To whom the Gaskell Prize (1887) was awarded.

2 To whom the Gaikell Privo (1889) was awrarded.

3 To whom the Gaskell Prizo (1890) was awardod.

4 To whom the Gakell Prise (1892) was arrarded.

5 To whom the Gaskell Prize (1895) was awrarded.

6 To whom the Gankell Prize (1896) was awardod.

7 To whom the Gaskell Prize (1897) was awrarded. 


\section{JOORNAL OF MENTAL SCIFNCE.}

\section{Acknowledgments. Exchange Journals.}

British.

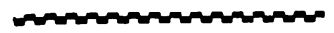

Brain ; British Medical Journal ; Dublin Medical Journal; Edinburgh Medical Journal; Glasgow Medical Journal ; Journal of the Anthropological Institute ; Hospital ; Lancet ; Mind ; Practitioner ; Proceedings of the Society for Psychical Research; Scottish Medical and Surgical Journal; Australasian Medical Gazette.

Americen.

Alienist and Neurologist; Journal of Insanity; Journal of Medical Sciences; Index Medicus; Journal of Comparative Neurology ; Journal of Mental and Nervous Diseases; Journal of Psychology; Medicine; Medico-Legal Journal; Medical Standard; Montreal Medical Journal; Psychological Review; Quarterly Journal of Inebriety ; Reports of the Smithsonian Institute; States Hospitals Bulletin, N.Y.; Transactions of the American Medico-Psychological Association; Universal Medical Journal.

\section{Erench.}

Annales Medico Psychologiques; Annales des Sciences Psychiques; Archives Clinicales de Bordeaux; Archives de Neurologie; Archives de Physiologie; Bulletin de Société Psychologie Physiologique ; Gazette des Hópitaux; Journal de Médicine de Bordeaux; Nouvelle Iconographie de la Salpêtrière; Polybiblion; Progress Módicale; Revue de Hypnotisme; Revue Philosophique; Revue Scientifique; Revue des Sciences Medicales; L'Anneo Psychologique.

Belgian.

Bulletin de Société de Médicine Mentale de Belgique.

German.

Allgemeine Zeitschrift für Psychiatrie; Archiv für Psychiatrio und Nervenkrankheiten; Centralblatt für Anthropologie; Centralblatt für Nervenheilkunde und Psychiatrie; Der Irrenfreund; Die Irrenpflege ; Jahrbucher für Psychologie; Neurologisches Centralblatt; Zeitschrift für Psychologie; Zeitschrift für Criminal Anthropologie ; Monatschrift für Psychiatrie und Neurologie.

Dutch.

Niederlandische Tijdschrift.

Italian.

Annali di Freniatria; Annali di Nevrologia; Il Manicomio Moderno; LAnomalo; La Psciatria; Rivista di Patologia nervosa e mentale; Rivista Sperimentale di Freniatria.

Enssian.

Archiv Psychiatrii, Nerrologuii, i Soudebnoi Psychopatologuii; Obozrenie Psychiatrii, Nevrologuii, i Experimentalnoi Psychologuii; Voprosi Nervno-psychitscheckoi Medizini : Voprosi philosophii i psyoholograi. 


\section{The Thyroid Treatment and the Preparations manufactured by ALLEN \& HANBURYS LTD.}

myxcedema. - The reliei affurded to cases of Myxcedema by the use of the Thyroid Glan during the last five years has marked a totally new departure in the hiotory of medicine. $T$ are beginning to understand the physiological importance of Internal Secretions to the anima oconomy.

Myrodema has been recognised as long ago as $187 \pm$ by Sir Wm. Gull as a distinct disease, and before that time it had been found that animals invariably succumbed after total extirpation
of the Thyroid.

Cretinism, too, had long been recognised to be connected with cougenital absence or deficiency in the glandular structure of the Thyroid.

Time enough has now elapsed to prove that those cases of MJxcedema which have been restored to hesith maintain that condition as long as they continue to take the gland or eficient prepuration of its extract.

Sporadic CretInIsm.-As regards Sporadic Cretinism, we cannot do better than refer the reader to an original article which appeareil in The Lancet, with Photographs, by W. W. O-, M.D. Oxon., M.R.C.P. London, November 4th, 1893, p. 1113.

All these cases were treated by Allen \& Hanbary' Thyroidin Tabelle. The results are perhaps more remarkable than those obtained in the allied condition of Myxoeilema. The writer observes that these preparations have given entire satisfaction.
The Photographs speak for themselves.

The success attending the u+e of Thyroid Extract in the myxcedematous condition of the skin and its appendages has suggested its employment in various skin lesions.

Psorlasis.- Very good results have been attained in some cases of obstinate psoriasis by using Thyroidin Tabelli.

In a recent number of The Lancet three of these cases are reported. After treatment with odide of potassium and arsenic internally, and chrysophanic acid ointment locally, showed no improvement, but, on the other hand, the disorder was in some cases aggrarated, developing

But on the Treatment being changed, and Tabelles of Thyrom Gland (Alles \& HasBuRYs') being administered, the writer observes : -

Crse 1. "The improvement was very striking . . the scales were completely gone, aud all that was left was a slight discoloration of the skin where the disease had appeared."

Case 2.-" The scalp and limbs were nearly clear. . . The patient, who has suffered from the disease for nineteen years past, has had no discomfort from the remedy, and says that nothing has ever acted so rapidly upon his disease."

Case 3.- "The result was astonishing, for in three days there was a marked improvement, and no further spread of the disease had taken place. No discomfort was experienced from the remedy."

For full details of the above quotations see The Lancet, January 6th, 1894, p. 19.

Whilst formerly liquid extracts and the raw gland were used, ALLFr \& HANBURYs were the firat to offer the active principles of the Thyroid Gland in the compressed form, which is now the favourite mode of administration. The glands are dissected and carefully examined and separated from all blood, fat, and inert tissue, which readily decompose and produce gastric disa most repulsive smeil, especialiy when they have been kept for a short time. Thyroidin Tabellø

Keadily disintegrate when swallowed, and from their shape and size are taken without difficulty If preferred, they may be dissolved in a little water before being swallowed.

\section{Thyroidin Cachots}

Contain 5 grs. of Thyroidin in each. Dose : 1 or 2. A very suitable and convenient mode of taking Thyroidin.

\section{Elizir Thyroidin}

Is a very palatable, slightly sweet preparation, of which one fluid ounce represents one Gland (average). It will be found to keep well without change. Dose: 1 to 4 fluid drachms.

The above are put up in 18., 2s. 6d., \& 48. 6d. packages (retail), or 8s., 218., \& 40 . per doz., to the Protession.

SAMPLES SUPPLIED TO THE PROFESSION ON APPLIOATION.

Allen and Hanburys Ltd., 年onghg Courd 
iv Journal of Mental Science, October. 1897.

Crown 8vo., with numerous Illustrations, price 4s.

\section{Mentally-Deficient Children :} 'IHEIR TREA'TMEN'T \& TRAINING.

By G. E. SHUTTLEWORTH, B.A., M.1)., \&c.

Late Medical Superintendent, Royal Albert Asylum for Idiots and Imbeciles of the Northern Countles, Lancaster; formerly Assistant Medical Officer, Earlswood Asylum.

" Altogether, there is no book on the subject so compact and so comprehensive, in which " the busy practitioner will find all he might care to know of the subject in a condensed but molentifio form. The book is well illustrated."-Edin. Med. Journal, Sept., 1893.

London: H. K. LEWIS, 136, Gower Street, W.C.

\section{The Journal of Nervous and Mental Disease.}

The Management announces the following arrangement of the staff for 1897 :Editors:

Dr. Chas. L. Dans, Dr. Philip Cuombs Knapp, Dr. Jas. J. Putnam, Dr. F. X. Dercam, Dr. Chas. K. Mills, Dr. M. Allen Starr. Dr. Philip Meirowitz. Associate Editors :

Managing Editor: Dr. Wm. G. Spiller.

Dr. Chas. Henry Brown, 25, West 45th Street, New York. To whom address all Editorial and Business Communications.

Price 3 Dollars per annum.

AMERICAN JOURNAL OF INSANITY.

Managing Editor-Dr. RICHARD DEWEY, Chicago.

Published Quarterly. Price five dollars per annum. Application to be made to Dr. Drwey, Venetian Buildings, Chicago.

\section{BULLETIN DE LA SOCIÉTÉ DE MÉDICINE MENTALE DE BELGIQUE.}

Quarterly Subscription, 10 franc8.-Apply to Dr. JULES MOREL, Médeoin en Chef de l'asile de Mons, Belginm.

\section{Gentralblatt}

FUR ANTHROPOLOGIE, ETHNOLOGIE, UND URGESCHICHTE. Editor, Dr. G. BUSCHAN.

A New Quarterly Journal, price 10 Marks per annum. It is designed to be a Periodical of an International character, and treats of the Sciences indicated by the Title.

J. U. KERN'S VerLAG (MaX MülleR) in Breslau.

All business communications relating to Advertisements to be addressed to Mr. J. H. Booty, 29, Wood Lane, Uxbridge Road, London, $W$. 


\section{Male Nurses' (TEMPERANGE) Co-operation. REMOVED TO}

10, THA YER STREET, MANCHESTER SQUARE, W.

Superior Trained MALE NURSEs for Medical, Burgical, Mental, Dipsomania, Fever, and Travelling Cases supplied at a moment's notice, Day or Night. All Nurses supplied by this Aseciation are TOTAL ABSTAINERB. BKILLED MASSECRS supplied. The only Temperance Asecciation of Male Nurses in the Kingdom. Terms : 1 guinea and a half or 2 guineas and upwardo per week. Nurses to receive their own fees. F. Bousk, Becretary.

Telegraphic Address : "Assuaged, London." Telephone 538, Paddington.

South Beacon, Hadlow Down, Buxted, Sussex.

A HOME FOR THE TEMPORARY CARE OF MENTAL A INVALIDS (Gentlemen only), requiring Treatment away from their own homes, but who are not ill enough to be certified.

Apply to-

PHILIP H. HARMER.

COMFORTABLE HOME in Country Rectory, for Lady or Gentleman (Invalid or otherwise).-Extensive gardens and grounds. Pony-chaise kept. Rooms occupied for many. years by Lady of Title. Healthy situation, within 50 miles of London. -Address, Rector, c/o Advertisement Manager, 29, Wood Lane, Uxbridge Road, London, W.

The Mental Affections of Children : Idiocy, Imbecility \& Insanity. By W. W. IRELAND.

$T_{Q}$ be published if two hundred subscribers are obtained. The book to contain about 300 pages octavo, with illustrations.

Price to subscribers Elght shllings.

Intending subscribers are requested to send their names to Dr. IreLaND, Mavisbush, Polton, Midlothian.

\section{TREATMENT OF INEBRIETY. \\ DUXHURST, REIGATE.}

Retreat for Ladies suffering from Inebriety and the abuse of Drugs. For particulars, apply to the Sister in charge.

Hope House, Hookwood, Horley, Retreat for middle-class Patients, Under the same Management as Duxhurst.

All business communications relating to Advertisements to be addressed to Mr. J. H. Booty, 29, Wood Lane, Uxbridge Road, London, $W$. 
vi Journal. uf Mentai. Science, Octobir, 1897.

\section{DAVID S. BRYSON, Victoria Oil, Paint, and Varnish Works, DUNDEE,}

Supplies the principal Asylums with Oils, Paints, Varnishes, Turpentine, Brushes, and Painters' Requisites of every description.

\section{FOR \\ IRON, BRASS, COPPER GOODS, LOCKS AND FITTINGS, In connection with Asylum and Hospital Work,}

\section{Apply BUSBY \& ROBERTSON,}

$$
\text { 56, ¿U.MILER ROW', BIRMINGHAM. }
$$

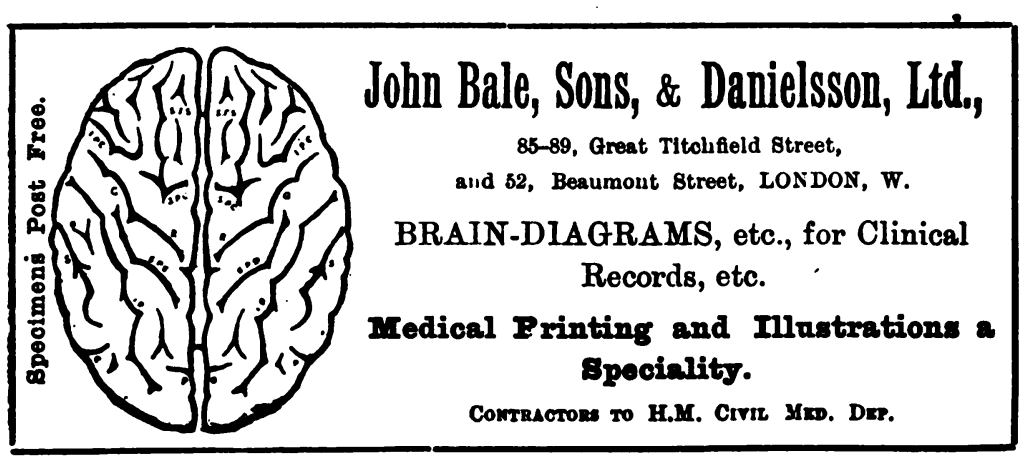

1l! business communications relating to Advertisements to be addressed to Alr. J. H. Booty, 29, Wood Lane, Uxbridge Road, London, W. 